\title{
Contribution à l'étude de la conformation du bétail local Rwanda de type Ankolé
}

\author{
par R. COMPÈRE \\ Chargé de Cours à I'Institut agronomique de l'Etat, d̀ Gembloux
}

\begin{abstract}
RÉSUMÉ
A partır des données rassemblées sur vingt-cinq années d'élevage au Centre zootechnique de Nyamiyaga (République Rwandaise), l'auteur a procédé à une étude approfondie de la conformation du bétail Rwandais de type Ankole. Portant sur seize mensurations prises régulièrement sur envıron quałre cents animaux mâles el femelles se situant dans la période de croissance, ces travaux précisent le rythme de développement des différentes parties du corps et proportions ; définissent le format standard du type de bétail étudié et fixent avec précision les objectı́s de l'amélioration de la conformation actuelle. En outre, les relations entre le poids vif et diverses mensurations ont été mises en équation, et des corrélations intéressantes ont été recherchées entre les principales grandeurs.
\end{abstract}

\section{INTRODUCTION}

L'étude approfondie de la conformation d'un bétail et plus spécialement d'un bétail à orientation boucherie tel que le local Rwanda est indispensable pour plusieurs rasons.

Tout d'abord, elle permet de compléter la connaissance des lois de la croissance des animaux, car dans bien des cas les seules données pondérales sont insuffisantes pour fixer l'état de développement des sujets, pour apprécier les défauts de conformation et pour définir d'une façon précise les variations avec l'âge des différentes parties du corps et proportions. L'étude de l'évolution des diverses mensurations chez les animaux en crossance facilite en outre l'établissement de plans rationnels en vue d'une sélection précoce.

La détermination d'un standard représente le second but de ces travaux. Ce standard sert de critère rigoureux pour l'élimination des individus ne possédant pas les caractéristiques moyennes du bétail et représente le document de base devant servir à la constitution d'un livre officiel pour l'inscription des reproducteurs.

Ensuite, l'étude de la dispersion des diverses mensurations tout en définissant les possibilités d'amélioration du cheptel, fixe les limıtes de format admises. Elle permet donc de'débarrasser la sélection d'un certain nombre d'estimations visuelles pas toujours très objectives ef d'orienter l'amélioration du type suivant des bases mathématiques.

Enfin, la détermination des corrélations et relations mathématiques existant, entre certaines grandeurs corporelles, diverses caractéristıques bouchères ef les rendements laitiers facilite grandement les travaux de sélection.

Grâce à ces relations, 11 est possible de diriger la sélection suivant des groupes de caractères ayant une liaison génétique, de profiter de l'indépendance existant entre deux séries d'observations pour repérer les sujets exceptionnels et de se baser sur les observations phénotypiques 
pour estimer la valeur bouchère et lattière des individus.

L'étude suivante a été réalisée au Centre d'Elevage de Nyamiyaga qui constitue pour le Rwanda la station officielle d'amélioration du bétail local. Les mensurations ont été effectuées sur la totalité des animaux présents dans le centre. Ceux-ci possèdent nécnmoins un degré plus ou moins grand d'amélioration qui leur confère à juste tıtre une conformation supérieure à celle du bétail élevé en mılıeu rural.

\section{DESCRIPTION DU TYPE DE BÉTAIL}

Le bétail du Rwanda appartient au type Ankole. Celui-ci englobe une série d'autres soustypes régionaux tels que le Bahema, le Bashi, l'Alur, etc., qui ont avec le bétail du Rwanda un ensemble de caractéristiques phénotypiques communes.

En outre, le type Ankole se range dans un ensemble plus large appelé "Groupe Sanga». Dans ce dernier sont rassemblés les différents types de bétail (Nilotic, Ankole, White Fulani, Rahaji, Jot Koram, etc.) qui dérivent du croisement ancien entre un taurin à longues cornes et un zébu à bosse cervico-thoracique bien marquée.

\section{Dispersion géographique}

L'aire de dispersion du type Ankole, de ses sous-types et de ses souches locales peut être représentée par un rectangle délimité par les $27^{\mathrm{e}}$ et $32^{\mathrm{e}}$ degrés de longitude Est, le $5^{\mathrm{e}}$ degré de latitude Sud et le $3^{\mathrm{e}}$ degré de latitude Nord.

Elle englobe les Etats ou parties d'Etats suivants :

- Uganda : une partie des Districts de Tori, de Kigezi et d'Ankole ;

- Tanganika : les Districts de Bokula, de Kibondo, de Kasulu ef de Ofipa :

- Royaume du Burundi ; République du Rwanda ;

- République du Congo : les territores de Bunia, d'Irumu et dans les provinces du Kivu : le District de Bukavu, les territoires de Rutshuru, de Masisi, d'Uvira, de Fizi et de Mwenga.

\section{Morphologie}

Les caractéristiques générales du bétaịl Ankole se résument comme suit :
- Anımal de grand format, à dos rectiligne, à bosse cervicale néanmaıns très discrète chez la femelle;

- Cornes longues et élancées ;

- Robe brun-rouge foncé mais aussi fréquemment rouge foncé, pie rouge, rouge clair, froment, rouan et rouge moucheté de blanc:

- Poids moyen à l'âge adulte de 350 à $400 \mathrm{~kg}$ pour les mâles et de $300 \mathrm{~kg}$ pour les femelles.

Il existe néanmoins de nombreux types spéciaux : à courtes cornes; sans cornes ; Bashi petit, à charpente fine et à cornes réduites; Rwanda à très longues cornes telles les anciennes vaches sacrées Inyambo.

\section{Mode d'entretien ef aptitudes du bétail étudié}

Les veaux demeurent en permanence avec les mères dans le troupeau jusqu'à l'âge de 8 mois et bénéficient de la totalité de la lactation. Toutefois, la production laitière de ce bétail est falble et varie entre 300 et 900 litres. Après le sevrage, its sont rassemblés en troupeaux d'une centaine de têtes pâturant nuit et jour les collines du centre d'élevage. Ces pâturages naturels non soumıs au régime des feux sont constitués par l'association végétale caractéristique de la régıon : Hyporrhenia filipendula et Brochiorio platynota.

Au cours de la saison des pluies de septembre à mai (1.000 à $1.100 \mathrm{~mm}$ ), l'herbe abondante produite par les pâturages constitue la totalité de la ration; pendant la période sèche de trois moss, le cheptel utilise la réserve de forn sur pied des bas-fonds ou reçoit une ration de 3 à $4 \mathrm{~kg}$ de silage de Pennisetum purpureum et de Brochiario ruzıziensis.

L'abreuvement se fait aux sources trois fois par jour.

La première saillie féconde a lieu en mayenne vers 1.100 jours et l'intervalle moyen entre deux vêlages s'élève à 473 jours.

\section{I. - FORMAT DE. LA TÊTE}

La tête est moyennement longue avec généralement une bonne largeur par rapport à so longueur et un mufle ample. Néonmoins, certains sujets possèdent une tête triangulaire étroite et longuement effilée, 'cette caractéristique correspond très souvent à une poitrine peu profonde et à un corps très étroit. 


\section{A. Mensurations.}

Deux mensurations ont été pratiquées, à savoir :

- la longueur totale de la tête, partant de la pointe du chignon à l'extrémité du mufle ;

- la largeur de la tête, prise à hauteur des tempes. Ces deux grandeurs ont été mesurées à l'aide du céphalomètre.

\section{B. Femelles.}

La forme de la tête des femelles ne constitue pas comme chez les géniteurs mâles un critère d'élimination. La tête courte, surtout en ce qui concerne la longueur du chanfrein, représente une beauté relative. On recherche néanmoins les sujets à front ef à mufle larges; un bon développement de ces régions caractérise, dit-on, un animal bon mangeur.

a) Développement de la tête (Tableau no 1).

Les études de ABERDOOS et BRODY ont démontré que la croissance des mensurations corporelles des animaux domestiques suit une loi générale de la forme :

$$
\log X=\log b+A \log K \text { ou } X=b e^{K A}
$$
où :

$X$ est la mensuration considérée,

A l'âge des animaux,

$b$ et $K$ des constantes,

e la base des logarithmes népériens.

Les équations donnant la longueur $« L_{t}$ » et la largeur « $I_{t}$ » de la tête exprimées en centimètres, en fonction de l'âge « $A$ » en mois sont :

Pour la longueur de la tête :

Du 1 er mols au $7 \mathrm{e}$ mois 3 jours :

$\log L_{t}=1,37140+0,02826 \mathrm{~A}$;

$$
L_{t}=23,52 \mathrm{e}^{0,06507 \mathrm{~A}}
$$

Du $7 \mathrm{e}$ mois 3 jours au $46 \mathrm{e}$ mois :

$\log L_{t}=1,54959+0,00316 \mathrm{~A}$;

$$
L_{t}=35,45 e^{0.00728 \mathrm{~A}}
$$

Pour la largeur de la tête:

Du $1 \mathrm{er}$ mols au $6 \mathrm{e}$ mols 3 jours :

$\log I_{t}=1,08680+0,01711 \mathrm{~A}$ :

$$
\mathrm{I}_{\mathrm{t}}=12,21 \mathrm{e}^{0.03940 \mathrm{~A}}
$$

Du 6 e mols 3 jours au $36 \mathrm{e}$ mois :

$$
\begin{array}{r}
\log l_{t}=1,17569+0,00257 \mathrm{~A} ; \\
I_{t}=14,98 \mathrm{e}^{\mathbf{0 . 0 0 5 9 2 A}}
\end{array}
$$

La croissance de la tête est pratiquement terminée à quatre ans. Le développement en largeur s'arrête entre 3 ans et 3,5 ans un peu plus tôt que celui en longueur qui se poursuit jusque 3,5 ans à 4 ans.

A Mulungu, la croissance de la tête jusqu'à l'âge de 4 ans a également été observée chez les demı-sang Bruns-Suisses (R. COMPËRE, 1960).

Au Soudan, J. PAGOT et R. DELAINE (1959) déterminent l'âge de 3 ans auquel les dimensions maxima de la tête des faurins N'Dama sont atteintes.

La tête croît plus en longueur qu'en largeur et le rapport entre ces deux dimensions se stabilise vers 4 ans, il passe de 1,98 à 2,55.

\section{b) Dimensions moyennes.}

Les dimensions moyennes de la tête des femelles adultes s'élèvent respectivement à $49,3 \mathrm{~cm}$ pour la-longueur et $19,2 \mathrm{~cm}$ pour la largeur.

\section{c) Dispersion des observations.}

Le tableau $n^{\circ} 5$ et les graphiques $n^{\circ} 3$ et no 4 renseignent sur la dispersion des observations à l'intérieur de la population des femelles adultes.

Les caractéristiques de l'échantillon observé sont :

Longueur de la tête :

váleur moyenne $\bar{X}: 49,3 \mathrm{~cm}$;

indice de dispersion $\sigma=1,51 \mathrm{~cm}$.

Largeur de la tête :

valeur moyenne $\bar{X}=19,2 \mathrm{~cm}$;

indice de dispersion $\sigma=0,99 \mathrm{~cm}$.

Le format recherché par la sélection sera le suivant :

- longueur totale de 47 à $49 \mathrm{~cm}$, soit $53,7 \%$ des observations ;

- largeur aux tempes de 20 à $23 \mathrm{~cm}$, soit $39,9 \%$ des observations.

La relation de dépendance existant entre ces deux grandeurs a été déterminée ci-après afin d'évaluer les chances en faveur de l'obtention rapide du format souhaité. 
Retour au menu

TABLEAD $n^{\circ} I$

Format des femelles (valeurs ajustées )

\begin{tabular}{|c|c|c|c|c|c|c|c|c|c|}
\hline \multirow{2}{*}{$\begin{array}{c}\text { en mois } \\
\text { ens }\end{array}$} & \multicolumn{2}{|c|}{ Dtmensions de la tête } & \multirow{2}{*}{$\begin{array}{l}\text { Longuetr } \\
\text { horizontele } \\
\text { du corps. }\end{array}$} & \multicolumn{5}{|c|}{ Différentes hauteurs. } & \multirow{2}{*}{$\begin{array}{l}\text { Périmètre du } \\
\text { métracarpe. }\end{array}$} \\
\hline & Langueur & $\begin{array}{l}\text { Lergeur } \\
\text { aux } \\
\text { tempes. }\end{array}$ & & Au gacrot. & $\mathrm{Au}$ dos. & Aur hanches & $\begin{array}{l}\text { A la pointe } \\
\text { des fesses. }\end{array}$ & $\begin{array}{l}\text { Du sternum } \\
\text { an sol }\end{array}$ & \\
\hline 1 & 25,1 & 12,7 & 70,6 & 79,0 & 78,4 & 85,2 & 72,3 & 49.5 & 12,5 \\
\hline 2 & 26,8 & 13,2 & 75,4 & 83,1 & 82,2 & 89,3 & 75,8 & 50,9 & 12,9 \\
\hline 4 & 30,5 & 14,3 & $\dot{8} 5,8$ & 91,8 & 90,5 & 98,1 & 83,2 & 53,7 & 13,8 \\
\hline 6 & 34,8 & 15,5 & 97,7 & 101,5 & 99,6 & 107.7 & 91,4 & 56.6 & 14.7 \\
\hline 12 & 38,4 & 16,1 & 102,8 & 107,7 & 104,4 & 111,8 & 97.3 & 60,1 & 15,8 \\
\hline 18 & 40,4 & 16.7 & 107,9 & 110,8 & 108,0 & 115,5 & 100,6 & 61,2 & 16,22 \\
\hline 24 & 42,2 & 17,3 & 113,4 & 114,0 & 111,8 & 119,3 & 104,0 & 62,3 & 16,7 \\
\hline 30 & 44,1 & 17,9 & 119.1 & 117,3 & 115,7 & 123,2 & 107,5 & 63,4 & $+7,1$ \\
\hline 36 & 46,1 & 18,5 & 125,1 & 120,7 & 119.7 & 127,2 & 111,1 & 64,5 & 17,6 \\
\hline
\end{tabular}

TABLEAU $n^{\circ}$ II

Format des femellos (suite)

\begin{tabular}{|c|c|c|c|c|c|c|c|c|c|}
\hline \multirow{2}{*}{$=\quad \begin{array}{c}\text { Age } \\
=\text { en mois }\end{array}$} & \multicolumn{3}{|c|}{ Dimensions de la poitrine } & \multirow{2}{*}{$\begin{array}{l}\text { Largeur } \\
\text { aux reins } \\
\text { - }\end{array}$} & \multicolumn{4}{|c|}{ Dimensions du bassin } & \multirow{2}{*}{$\begin{array}{l}\text { Poids } \\
\text { moyen } \\
\text { en kg }\end{array}$} \\
\hline & Largeur & Profondeur & $\begin{array}{l}\text { Périmètre } \\
\text { thoracique. }\end{array}$ & & $\begin{array}{c}\text { Largeur } \\
\text { aux hanches }\end{array}$ & $\begin{array}{c}\text { Largeur aux } \\
\text { articulations } \\
\text { coxo-fémoralès }\end{array}$ & $\begin{array}{l}\text { Largeur à } \\
\text { la pointe } \\
\text { des fesses. }\end{array}$ & $\begin{array}{c}\text { Longueur } \\
\text { du } \\
\text { bassin }\end{array}$ & \\
\hline 1 & 17,2 & 30,5 & 96,2 & 14,5 & 18,4 & 20,5 & 12,9 & 24,5 & 49 \\
\hline 2 & 18,1 & 32,8 & 103,0 & 15.5 & 20,0 & 21,7 & 13,5 & 26,1 & 58 \\
\hline 4 & 20,2 & 37,8 & 116,5 & 17.8 & 23,6 & 24,5 & 14,9 & 30,0 & 86 \\
\hline 6 & 22,5 & 43,6 & 131,6 & 20,4 & 27,9 & 27.6 & 16,4 & 34,4 & 127 \\
\hline 12 & 24,0 & 46,0 & 137,9 & 22,7 & 30,4 & 29,6 & 16,8 & 34,9 & 150 \\
\hline 18 & 25.6 & 48,6 & 144,5 & 24,3 & 32,4 & 30,9 & 17,8 & 37,0 & 176 \\
\hline 24 & 27,2 & 51,4 & 151,5 & 26,0 & 34,6 & 32,4 & 18,8 & 39,1 & 226 \\
\hline 30 & 29,0 & 54.3 & 158,7 & 27,8 & 37,0 & 33,8 & 20,0 & 41,5 & 268 \\
\hline 36 & 30,9 & 57,4 & 166,4 & 29,8 & 39,6 & 35,4 & 21,2 & 43,9 & 310 \\
\hline
\end{tabular}


TABLEEAU $n^{0}$ III

Format des mâles (valourg ajustées)

\begin{tabular}{|c|c|c|c|c|c|c|c|c|c|}
\hline \multirow{2}{*}{ en moio } & \multicolumn{2}{|c|}{ Dimensions de la tête } & \multirow{2}{*}{$\begin{array}{l}\text { Longueur } \\
\text { horizontalo } \\
\text { du corps }\end{array}$} & \multicolumn{5}{|c|}{ Différentes hautours. } & \multirow{2}{*}{$\begin{array}{l}\text { Périmètre } \\
\quad \text { du } \\
\text { métacarpe }\end{array}$} \\
\hline & Longueur & $\begin{array}{l}\text { Largour } \\
\text { aux } \\
\text { tompes. }\end{array}$ & & Au garrot. & Au dos. & Aux hanches & $\begin{array}{l}\text { A la pointe } \\
\text { des fesses }\end{array}$ & $\begin{array}{l}\text { Dus atermum } \\
\text { su sol }\end{array}$ & \\
\hline 1 & 25.5 & 13,1 & 72,5 & 80,7 & 79,2 & 89,7 & 72,8 & 49,9 & 13,2 \\
\hline 2 & 27,2 & 13,7 & 77,3 & 84,8 & 83,3 & 93,1 & 76,3 & 51,5 & 13,6 \\
\hline 4 & 31,1 & 15,0 & 887,9 & 93,6 & 92,1 & 100,3 & 83,8 & 54,8 & 14,6 \\
\hline 6 & 35.5 & 16,4 & 100,0 & 103.3 & 101,8 & 108,0 & 92,1 & 58,4 & 15,6 \\
\hline Taureaux & & & & & & & & & \\
\hline 18 & 43,7 & 19,6 & 118,1 & 119,8 & 118,0 & 123,5 & 107,9 & 65.1 & 17,8 \\
\hline 24 & 45,7 & 20.1 & 124,5 & 123,8 & 121,7 & 130,4 & 110,9 & 66,2 & 18,6 \\
\hline 30. & 47,8 & 20,6 & 131,3 & 127,9 & 125,5 & 134,5 & 113,9 & 67,2 & 19,4 \\
\hline 36 & 50,1 & 21,2 & 138,5 & 132,1 & 129,4 & 138,7 & 117,1 & 68,3 & 20,3 \\
\hline Bosufs & & - & & & & & & & \\
\hline 18 & 41,3 & 17,9 & 110,4 & · 11 & 111,2 & 118,8 & 104,5 & 61,8 & 17,1 \\
\hline 24 & 43,2 & 18,3 & 115,8 & 115,4 & 114,7 & 122,6 & 107,3 & 63.1 & 17,8 \\
\hline 30 & 45,1 & 18,8 & 121,4 & 119,3 & 118,3 & 126.5 & 110,2 & 64.5 & 18,5 \\
\hline 36 & 47,2 & 19,2 & 127,3 & $123,4^{\circ}$ & 122,1 & 130,5 & 113,2 & 65,8 & 19,2 \\
\hline
\end{tabular}


TABLEATI No IV

- Format des malles -

(suite)

\begin{tabular}{|c|c|c|c|c|c|c|c|c|c|}
\hline & \multicolumn{2}{|c|}{ Dimensions de la poitrine } & & & & \multicolumn{2}{|c|}{ Dimensions du bassin } & \multirow[b]{2}{*}{$\begin{array}{l}\text { Longueur } \\
\text { bassin }\end{array}$} & \multirow{2}{*}{$\begin{array}{l}\text { Poids moyen } \\
\text { en kg }\end{array}$} \\
\hline Age & Largeur & Profondeur & $\begin{array}{l}\text { Pórimètre } \\
\text { thoractque }\end{array}$ & $\begin{array}{l}\text { Largeur alux } \\
\text { reins }\end{array}$ & $\begin{array}{c}\text { Largeur aux } \\
\text { hanches }\end{array}$ & $\begin{array}{l}\text { Largeur aux } \\
\text { arttculations } \\
\text { exo-fómorales }\end{array}$ & $\begin{array}{l}\text { Largour à } \\
\text { la pointe } \\
\text { des fosses }\end{array}$ & & \\
\hline 1 & 18,0 & 30,8 & 97,4 & 14,4 & 18,2 & 20,9 & 12,2 & 24,8 & 45,3 \\
\hline 2 & 19,0 & 33,3 & 104,5 & 15,6 & 19,8 & 22,2 & 13,1 & 26,6 & 67,8 \\
\hline 4 & 21,1 & 38,7 & 120,3 & 18,2 & 23,4 & 24,9 & 15,0 & 30,6 & 99,4 \\
\hline 6 & 23,5 & 45,0 & 138,5 & 21,3 & 27,8 & 28,0 & 17,2 & 35.2 & 134,6 \\
\hline$\frac{\text { Taureaux }}{18}$ & 28,2 & 54,7 & 161,3 & 27,1 & 35,4 & 33,6 & 20,2 & 41,6 & 264,0 \\
\hline 24 & 30,4 & 57,6 & 169,9 & 29,2 & 37,9 & 35,2 & 21,7 & 43,9 & 350,0 \\
\hline 30 & 32,8 & 60,6 & 181,9 & 31,4 & 40,6 & 37,0 & 23.4 & 46.3 & 397,5 \\
\hline 36 & 35,4 & 63,7 & 188,6 & 33,7 & 43,5 & 38,8 & 25.1 & 48,8 & 443,0 \\
\hline $\begin{array}{r}\text { Boevfs } \\
18\end{array}$ & 25,6 & 51,2 & 150,0 & 24,9 & 32,6 & 32,2 & 18,8 & 37,9 & 192,0 \\
\hline 24 & 26,9 & 53,5 & 155.9 & 26,5 & 34,5 & 33,4 & 19,6 & 39.8 & 253,0 \\
\hline 30 & 28,3 & 55,9 & 161,9 & 28,2 & 36.5 & 34,5 & 20,5 & 41,7 & 266,0 \\
\hline 36 & 29,8 & 58,3 & 168,2 & 30,0 & 38,7 & 35,7 & 21,3 & 43,8 & 283,0 \\
\hline
\end{tabular}




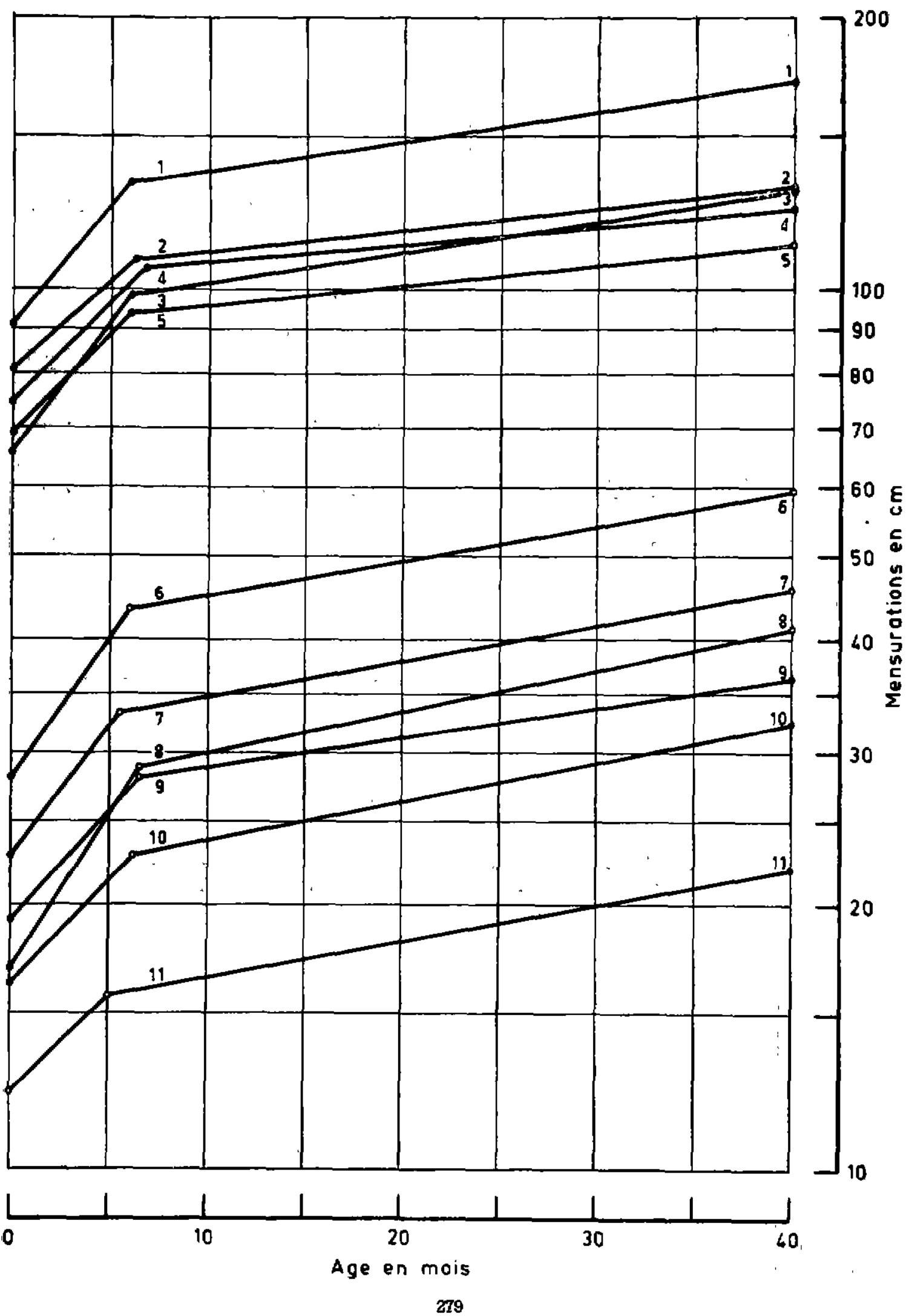




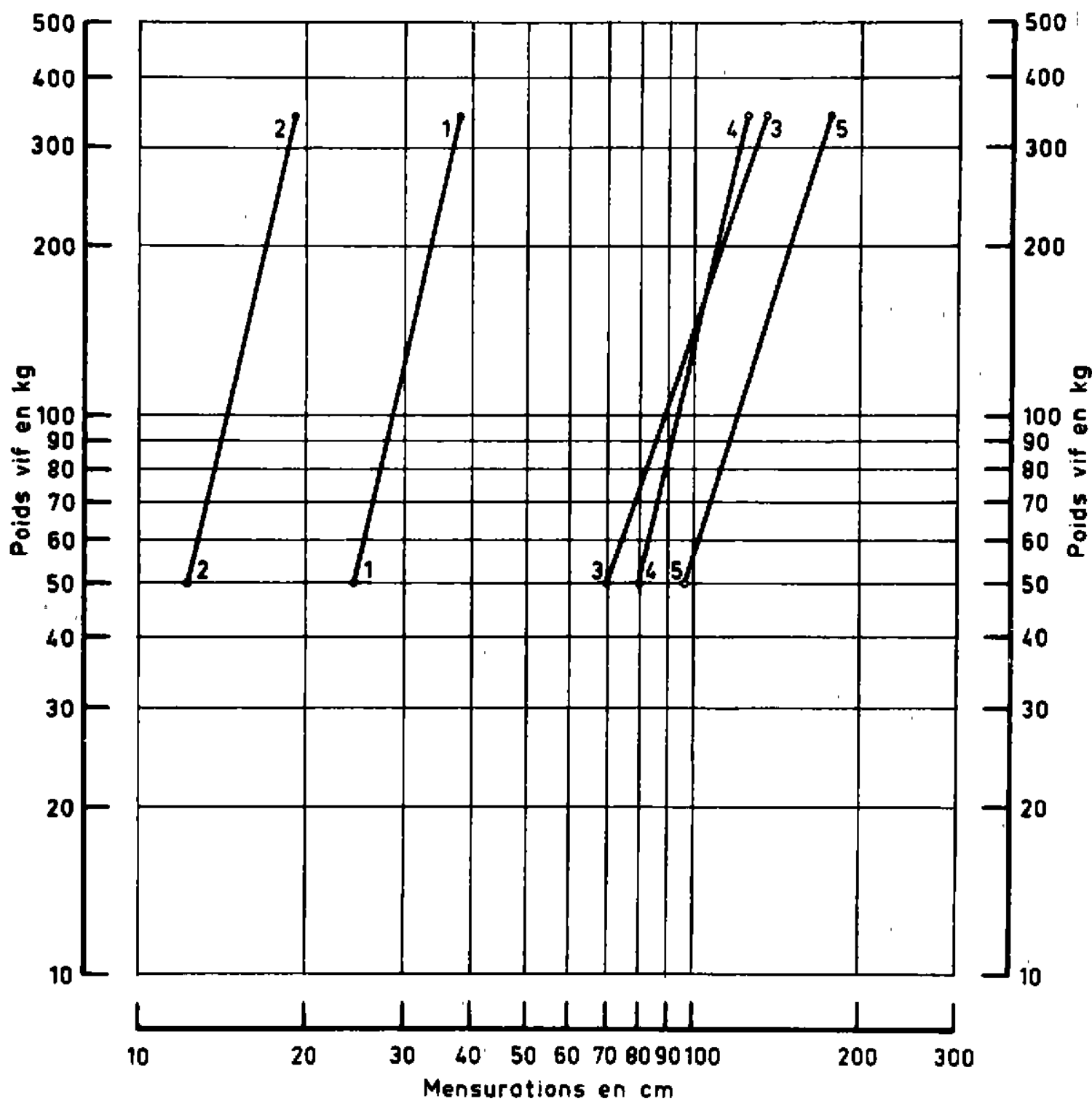

GRAPHIQUE no 1. - Croissance absolue des femelles.
(1) Périmètre thoracique.
(2) Hauteur aux hanches.
(3) Longueur du corps.
(4) Hauteur au dos.
(5) Hauteur à la pointe des fesses.
(6) Profondeur de poitrine.
(7) Longueur du bassin.
(8) Largeur aux hanches.
(9) Largeur aux articulations coxo-fémorales.
(10) Largeur de poitrine.
(11) Largeur à la pointe des fesses.

GRAPHIQUE no 2. - Croissance relativé du poids des femelles en fonction de :

(1) Lo longueur de la tête.

(2) La largeur de la tête.

(3) La longueur du cotrps

(4) La hauteur au garrot.

(5) Le périmètre thoracique. 


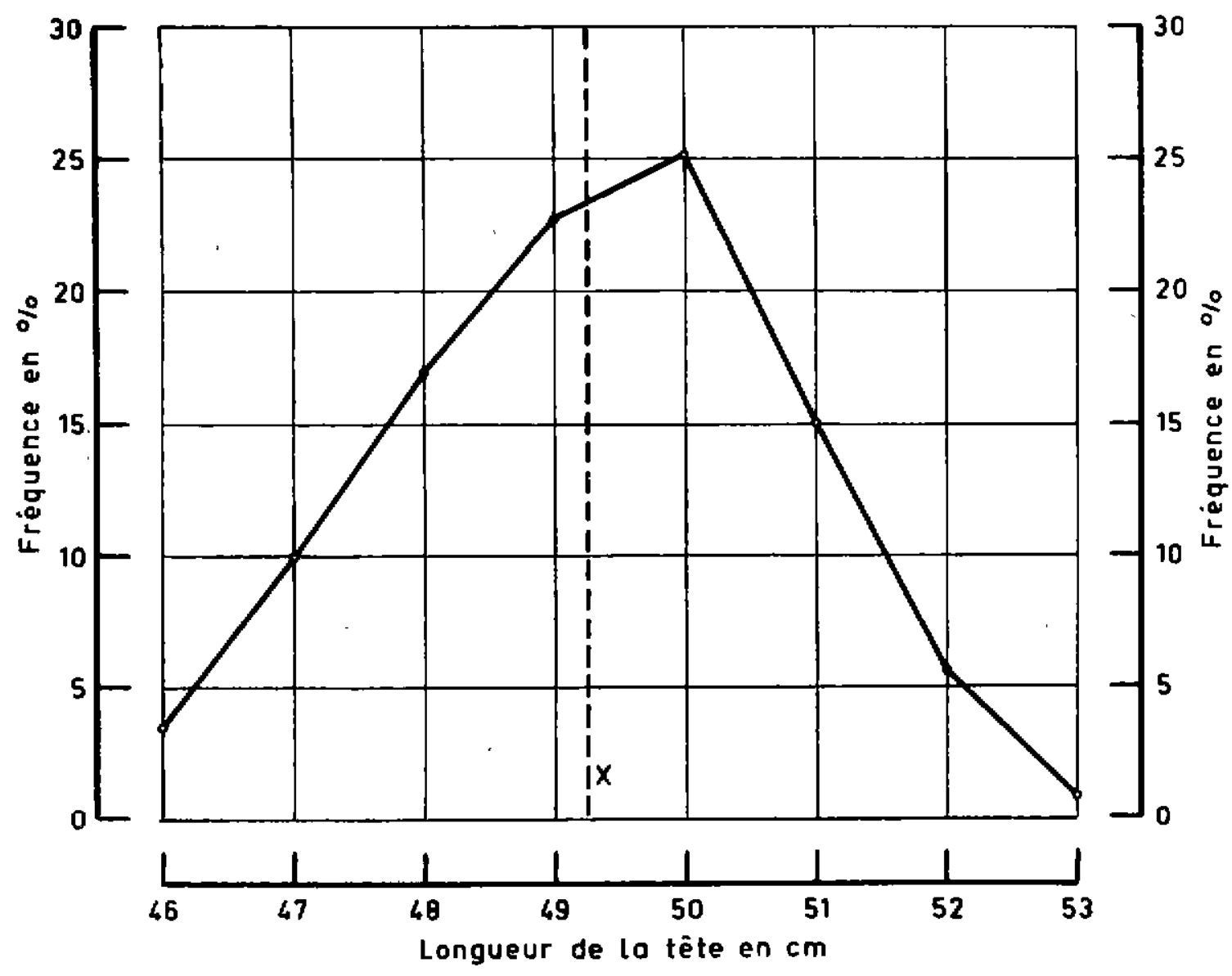

GRAPHIQUE n 3. - Polygone de fréquence des longueurs de la tête des femelles.

d) Relation entre les observations.

\section{Animaux en croissance.}

Croissance relative du poids vif et de la longueur de la tête (graphique no 2 ).

Le coefficient de corrélation entre d'une part le poids vif et d'autre part la longueur de la tête d'un même individu s'élève à $+0,97 \times x \times$ $+0,78$ (valeur limite du coefficient de corrélation au seuil $P=0,01)$.

La relation entre ces deux grandeurs est définie par l'équation : ou

$$
\text { - } \log \text { Poids }=2,863.30 \log L_{t}-2,30685
$$

$$
\text { Poids }=0,00493 L_{t}{ }^{2,86}
$$

Croissance relative au poids vif et de la largeur de lo tête (graphique $n^{\circ} 2$ ).

Le coefficient de corrélation, à peu près semblable au précédent, atteint $+0,98$ et la relation est représentée par la formule :

$$
\log \text { Poids }=4,660.61 \log I_{t}-3,43166
$$
ou

$$
\text { Poids }=0,\left.000370\right|_{t} ^{\mathbf{4 , 6 6}}
$$

\section{Femelles aduifes.}

Corrélation entre la longueur ef la largeur de la tête.

Afin de définir le degré de dépendance pouvant exister entre ces deux mensurations, le 


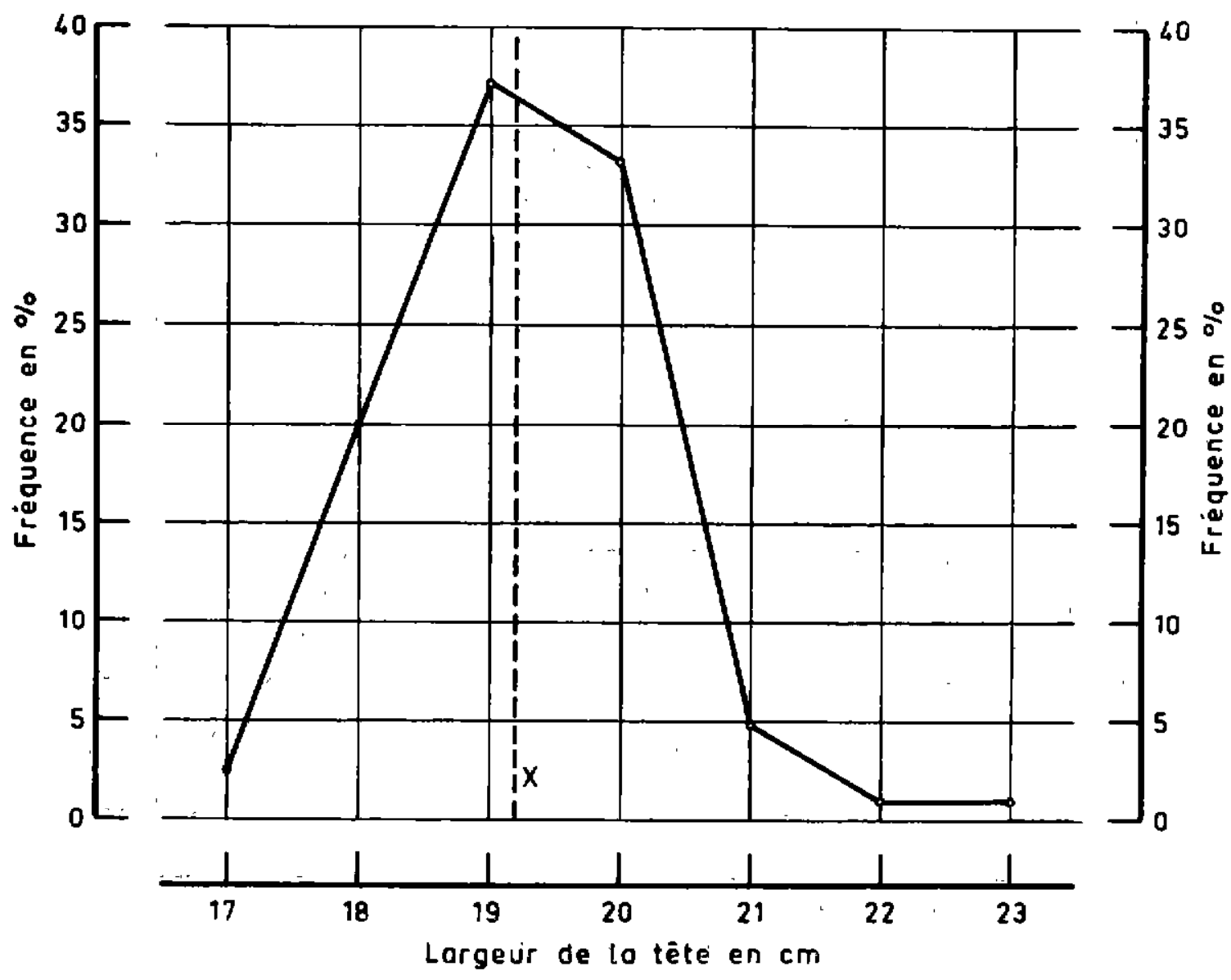

GRAPHIQUE no 4. - Polygone de fréquence des largeurs de tête des femelles.

TABIEAD $n^{\circ} \nabla$

Digpergion des donréen individuelles

\begin{tabular}{|c|c|c|c|c|}
\hline \multicolumn{2}{|c|}{ Longuevr de la tote } & \multicolumn{3}{|c|}{ Largeur de la tete } \\
\hline Dinensious (am) & Fréquence en p.100 & Dimengions ( $\mathrm{cm}$ & Fréquence en & p. 100 \\
\hline 46 & 3,5 & 17 & 2,6 & : \\
\hline 47 & 10,1 & 18 & 20,2 & \\
\hline 48 & 17,2 & 19 & 37,3 & \\
\hline 49 & 22,9 & 20 & 33,5 & $\cdot$ \\
\hline 50 & 25,1 & 21 & 4,7 & \\
\hline 51 & 15,0 & 22 & 0,9 & \\
\hline 52 & 5,3 & 23 & 0,8 & \\
\hline 53 & 0,9 & & & \\
\hline
\end{tabular}


coefficient de corrélation a été calculé sur la population des femelles adultes. II s'élève à $-0,57 \mathrm{xx}>-0,32.1$ (valeur limite du coefficien de corrélation au seuil $P: 0,001$ ). Une corrélation négative hautement significative existe donc entre les deux variables, ce qui signifie qu'til est possible de sélectionner assez aisément des sujets à tête courte et large. Cette relation est actuellement exploitée avec profit par. les différentes méthodes de sélection.

\section{Les MALes.}

Le nombre de données étant beaucoup moins grand pour' les mâles que pour les femelies, vu qu'ilš sont sacrifiés' assez tôt pour la boucherie, on se limitera à dés conclusions et à des considérations beaucoup plus générales. A partir de l'âge de 1 an, les taureaux et les b́ceufs ont été groupés séparément.

Les mâles possèdent une tête plus longue et plus large que les femelles.

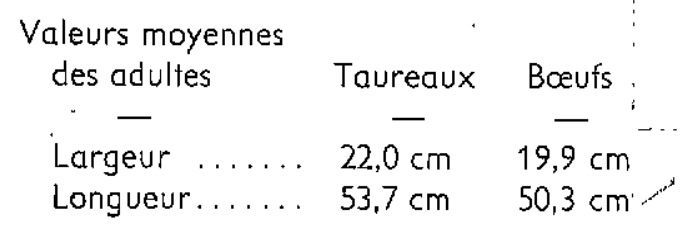

Le rapport longueur/largeur est à peu près semblable chez les bceufs ef les femelles tandis que les taureaux reproducteurs possèdent une tête plus massive.

Les équations donnant les dimensions de la tête « $L_{t}$ et $I_{t}$ 》 exprimées en centimètres en fonction de l'âge «A » en mois, sont :

Pour la longueur de la tête :

- Du 1er mois au 6 e mois :

$\log L_{t}=1,37701+0,02876 \mathrm{~A}$ :

$L_{t}=23,82 \mathrm{e}^{0,06622 \mathrm{~A}}$.

- Au-delà du 12e mors chez les taureaux : $\log L_{t}=1.58058+0.00330 \mathrm{~A}$ :

$$
\mathrm{L}_{\mathrm{t}}=38,07 \mathrm{e}^{0,00760 \mathrm{~A}}
$$

- Au-delà du 12e mols chez les boufs $\log L_{t}=1,55822+0,00321 A$;

$$
L_{t}=36,16 \mathrm{e}^{0.00730 \mathrm{~A}}
$$

Pour la largeur de la tête:

- Du 1 er mois au 6 e mols :

$\log I_{t}=1,09607+0,01969 \mathrm{~A}$;

$l_{t}=12,48 \mathrm{e}^{0,04534} \mathrm{~A}$.
- Au-delà du $12^{\mathrm{e}}$ mois chez les taureaux :

$\log I_{\mathbf{t}}=1,25969+0,00183 \mathrm{~A}$;

$$
l_{t}=18,18 e^{0,00421 A}
$$

- Au-delà du 12e mois chez les bœufs:":

$\log l_{t}=1,22243+0,00169 A$

$$
\mathrm{l}_{\mathrm{t}}=16,69 \mathrm{e}^{0,00389 \mathrm{~A}}
$$

\section{I1. - LONGUEUR DU CORPS}

Le local Rwanda possède une longueur du corps très satısfaisante. Elle tend néanmoins à être augmentée par la sélection si toutefois elle s'allie avec une profondeur de poitrine suffisante.

\section{A. mensuration.}

La longueur horizontale du corps est mesurée depuis l'extrémité antérieure du sternum jusqu'à la pointe des fesses. Cette distance est prise en projection au moyen des deux tiges articulées de la toise.

\section{B. LES fEMELLES.}

Un bon développement en longueur des femelles est une caractéristique recherchée. Les animaux courts sont généralement très légers et déficients en ce qui concerne de nombreux autres caractères phénotypiques; par contre, une longueur du corps excessive prodult des anımaux efflanqués et à musculature réduite.

\section{a) Développement en longueur.}

Le tableau no 1 et le graphique $n^{0} 1$ schématisent la croissance en longueur de la population des femelles.

La crolssance en longueur est pratiquement terminée à l'âge de 4 ans à 4 ans et demı. A Mulungu (COMPËRE, R., 1960) la croissance en longueur des demi-sang Bruns-Suisses se poursult jusqu'à 4 ans et demi ò 5 ans tandis que pour le N'Dama, J. PAGOT et R. DELAINE' (1959) signalent l'arrêt de la croissance entre 2; ans et 2 ans et demı.

Les équations qui donnent la longueur hor-

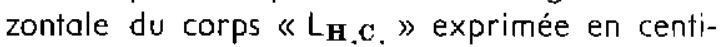
mètres, en fonction de l'âge « $A$ » en mols, sont :

Du 1 er mois au 6e mols 1 jour :

Log L $_{\text {H.C. }}=1,82088+0,02818 \mathrm{~A}$ :'

$$
\mathrm{L}_{\text {H.C. }}=66,20 \mathrm{e}^{0,06489 \mathrm{~A}}
$$

Du 6e mois 1 jour au 46e mols :

$\log L_{H . C}=1,96913+0,00356 \mathrm{~A}$ :

$$
\mathrm{L}_{\text {H.C. }}=93,14 \mathrm{e}^{0,00820 \mathrm{~A}}
$$


Le corps croît plus en longueur qu'en hauteur. Une modification de la forme générale du corps est observée de la façon suivante au cours de la croissance :

- plus haut que long jusqu'à 2 ans ;

- de forme carrée de 2 ans à 2 ans et demi ;

- plus long que hautà partir de 2 ans et demi.

b) Valeur moyenne.

La longueur moyenne du corps des femelles adultes est de $135,4 \mathrm{~cm}$. Pour le bétail de Luvironza (Burundi) P. MATHIEU (A. FOCAN, 1959) signale une valeur moyenne de $134,2 \mathrm{~cm}$. A. Nioka, M. MARICZ (1961) fournit, pour les sous-types Alur et Bahema (même type Ankolé que le local Rwanda) les dimensions suivantes:

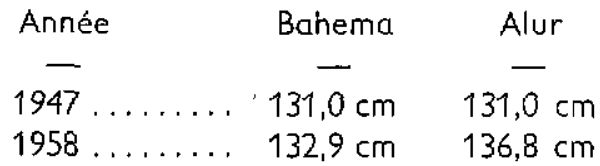

c) Dispersion des observations.

Le tableau $n^{\circ} 6$ et le graphique no 5 illustrent la dispersıon des observations à l'intérieur de la population femelle adulte.

Les caractéristiques de l'échantillon observé à Nyamiyaga sont :

Valeur moyenne $\bar{X}=135,4 \mathrm{~cm}$.

Indice de dispersion $\sigma=5,58 \mathrm{~cm}$.

La longueur recherchée par la sélection en rapport avec des proportions normales du corps se situe entre 136 et $143 \mathrm{~cm}$ ce qui représente $54,5 \%$ des observations.

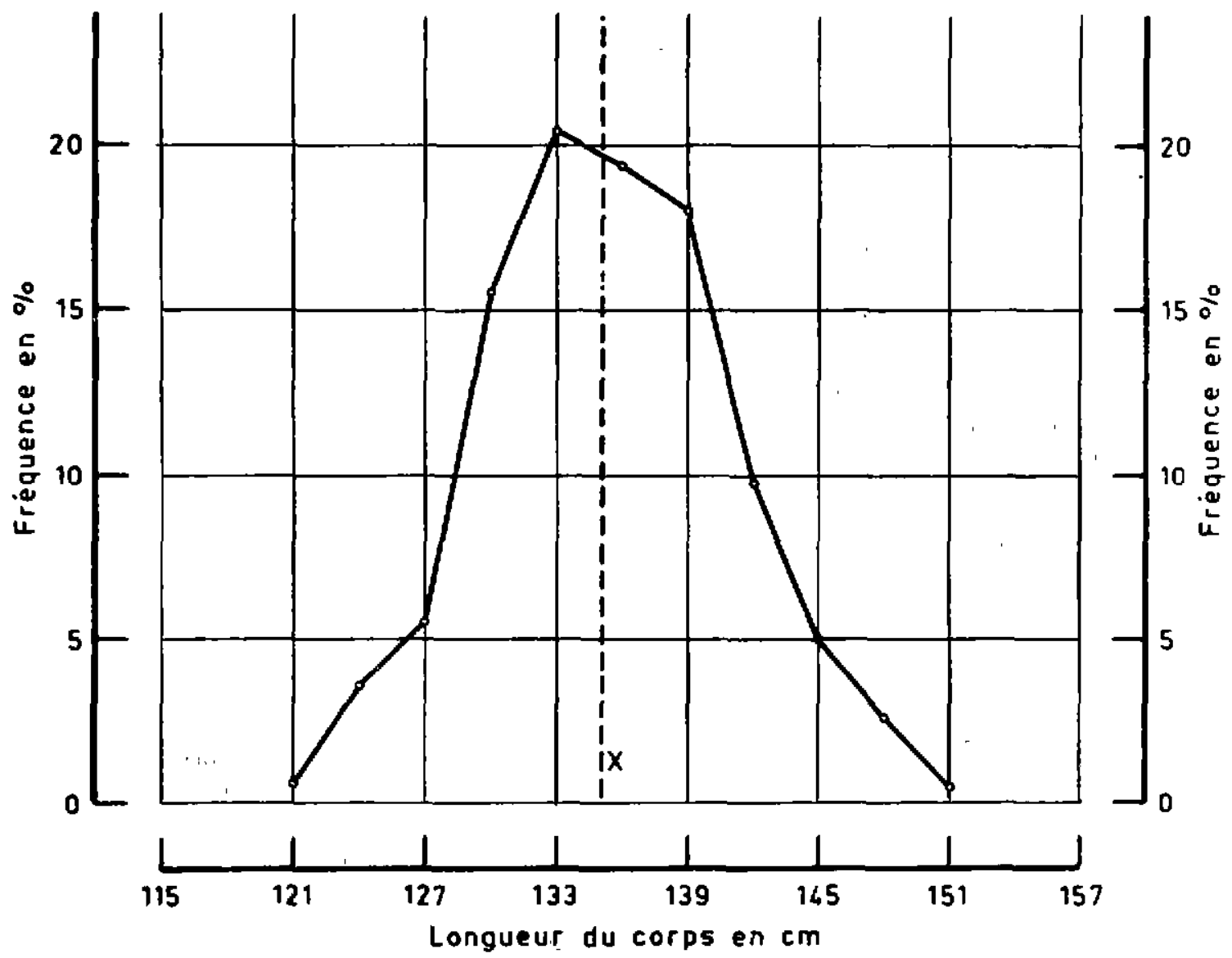

GRAPHIQUE no 5. - - Polygone de fréquence des longueurs horizontales du corps chez les femelles. 
TABLEAUT IN VI

Bispersion des données individuellos

\begin{tabular}{|c|c|c|c|}
\hline $\begin{array}{c}\text { Longueur } \\
\text { du corps } \\
\text { en cm }\end{array}$ & $\begin{array}{c}\text { Frequence } \\
\text { en g.100 }\end{array}$ & $\begin{array}{c}\text { Hauteur } \\
\text { au garrot } \\
\text { en cm }\end{array}$ & $\begin{array}{c}\text { Fréquence } \\
\text { en p. 100 }\end{array}$ \\
\hline 121 & 0,5 & 117 & 1,3 \\
124 & 3,5 & 120 & 6,9 \\
127 & 5,4 & 123 & 19,0 \\
130 & 15,5 & 126 & 37,5 \\
133 & $20,4-$ & 129 & 23,3 \\
136 & 19,4 & 132 & 9,1 \\
139 & 18,0 & 135 & 1,7 \\
142 & 9,7 & 138 & 0,4 \\
145 & 4,9 & & \\
140 & 2,5 & & \\
151 & 0,5 & & \\
\hline
\end{tabular}

\section{d) Relations entre les observations.}

\section{Animaux en croissance.}

Croissance relative du poids vif ef de la longueur horizontale du corps (graphique $n^{\circ} 2$ ).

Le coefficient de corrélation entre le poids vif et la longueur du corps s'élève à $+0,96$, valeur hautement significative.

La relation entre ces deux variables est représentée par l'équation :

$$
\begin{aligned}
& \text { Log Poids }=2,82897 \log L_{\text {H.C. }}-3,51871 \\
& \text { ou } \\
& \text { Poids }=0,000303 L_{\text {H.C. }}{ }^{2,82}
\end{aligned}
$$

Chez le bétail N'Dama, J. PAGOT et R. DELAINE obtiennent une relation semblable :

$$
\text { Poids }=0,000825 \mathrm{~L}_{\text {H.C. }},{ }^{2,52}
$$

\section{Femelles adultes.}

Corrélation entre la langueur horizontale du corps et la hauteur au garrot.

Pour l'ensemble des données recueillies chez les femelles adulies, il existe une corrélation positive hautement significative entre la longueur du corps ef la hauteur au garrot.

$$
\begin{gathered}
r\left(L_{H}, \mathrm{c} .\right. \\
\text { (Au seull } P: 0,001)
\end{gathered}
$$

Il sera donc malaisé de dissocier ces deux caractères et, dans le cas des objectifs de la sélection envisagés jadis à Nyamiyaga, d'obtenir des animaux longs et à la fois très rapprochés du sol.

\section{LES MÂLES.}

Les mâles sont plus longs que les femelles

$$
\text { Age moyen }
$$

Longueur moyenne en $\mathrm{cm}$

Taureaux

\section{7 mois}

Bœufs 45 mois 21 jours

133,8

Les proportions générales du corps sont à peu près identiques d̀ celles des femelles.

Les équations donnant la longueur du corps, exprimée en centimètres, en fonction de l'âge en mois, sont:

Du 1 er au $6 \mathrm{e}$ mois :

$$
\begin{gathered}
\log L_{\text {H.C. }}=1,83215+0,02797 \mathrm{~A} \text {; } \\
L_{\text {H.C. }}=67,94 \mathrm{e}^{0,06440 \mathrm{~A}}
\end{gathered}
$$

Au-delà du $12^{\mathrm{e}}$ mois chez les taureaux :

$$
\begin{gathered}
\log L_{\text {H.C. }}=2,00310+0,00384 \mathrm{~A} \text {; } \\
L_{\text {H.C. }}=100,70 \mathrm{e}^{\mathbf{0}, 00884 \mathrm{~A}}
\end{gathered}
$$

Au-delà du $12^{\mathrm{e}}$ mois chez les boufs :

$$
\begin{gathered}
\log L_{\text {H.c. }}=1,98078+0,00345 \mathrm{~A} \text {; } \\
L_{\text {H.C. }}=95,67 \mathrm{e}^{\mathbf{0 . 0 0 7 8 4 A}}
\end{gathered}
$$

\section{III. - DÉVELOPPEMENT DE LA TAILLE}

Le bétail local du Rwanda présente généralement un développement en hauteur excessif par rapport à la profondeur du corps. La ligne du dos est légèrement inclinée vers l'avant tandis que la lıgne de croupe possède l'inclınaison prononcée du bétal de boucherie qu mixte.

\section{A. Mensurations.}

Les quatre mensurations suivantes ont été prises en considération :

- la hauteur au garrot : du sol à l'extrémité postérieure de la bosse cervicale,

- la hauteur au dos : du sol à l'épi dorsal,

- la hauteur aux hanches : du sol à la ligne qu réunit les deux hanches:

- la hauteur à la pointe des fesses : du sol aux tubérosités ischiatiques.

\section{B. LES FEMELLES.}

Les bonnes femelles ont en général une taille supérieure à la moyenne qui s'accompagne d'une inclinaison moyenne du dos et de la croupe. 


\section{a) Développement de la taille.}

Le tableau no 1 et le graphique no 1 permettent de suivre la croissance de la taille des femelles.

La croissance en hauteur du train antérieur : hauteur au garrot et hauteur au dos se poursuit jusqu'à 3 ans à 3 ans et demi, tandis que celle du train postérieur : hauteur aux hanches et à la pointe des fesses se termıne un peu plus tard entre 3 ans et demi et 4 ans.

A Mulungu (COMPÉRE, R., 1960) la croissance en hauteur du'demi-sang Brun-Suisse se termine entre 4 ans et 4 ans et demi tandis que J. PAGOT et col. (1959) signalent une croissance encore beaucoup plus longue chez le bétail N'Dama où la limite se situe vers 5 ans.

Les équations donnant les dimensions en hauteur : hauteur au garrot $\ll \mathrm{H}_{\mathrm{g}} »$, hauteur au dos 《 $H_{d} »$, hauteur aux hanches « $H_{h} »$, hauteur ò la pointe des fesses « $H_{f}$ », distance du sternum au sol « $D_{\text {st.s }}$ » en centımètres, en fonction de l'âge en mois, sont:

\section{Pour la hauteur au garrot :}

du 1 er mois au 6 e mois 20 jours:

$$
\begin{gathered}
\log H_{g}=1,875.77+0,02181 \mathrm{~A} ; \\
H_{g}=75,12 \mathrm{e}^{0,05022 \mathrm{~A}}
\end{gathered}
$$

du $6 \mathrm{e}$ mois 20 jours au $36^{\mathrm{e}}$ mois :

$$
\begin{gathered}
\log H_{g}=2,00764+0,00206 \mathrm{~A}: \\
H_{\underline{y}}=101,8 \mathrm{e}^{0.00474 \mathrm{~A}}
\end{gathered}
$$

Pour la hauteur au dos:

du 1 er mois au $6 \mathrm{e}$ mois 9 jours :

$$
\begin{gathered}
\log H_{\mathbf{d}}=1,87326+0,02085 \mathrm{~A} \text {; } \\
\mathrm{H}_{\mathbf{d}}=7,4,69 \mathrm{e}^{\mathbf{0 . 0 4 8 0 1} \mathrm{A}}
\end{gathered}
$$

du 6e mols 9 jours au 36e mois :

$$
\begin{gathered}
\log H_{\mathbf{d}}=1,98911+0,00247 \mathrm{~A}: \\
H_{\mathbf{d}}=97,52 \mathrm{e}^{\mathbf{0} 00569 \mathrm{~A}}
\end{gathered}
$$

Pour' la hauteur aux hanches: du 1 er mois au 6 e mors 4 jours :

$$
\begin{gathered}
\log H_{h}=1,91018+0,02034 A ; \\
H_{h}=81,32 \mathrm{e}^{0,04883 \mathrm{~A}}
\end{gathered}
$$

du $6 \mathrm{e}$ mois 4 jours au $46^{\mathrm{e}}$ mois :

$$
\begin{gathered}
\log H_{h}=2,02067+0,00233 A ; \\
H_{h}=104,9 e^{0,00537 A}
\end{gathered}
$$

Pour la distance, du sternum au sol : du 1 er mois au 7 e mois 15 jours:

$$
\begin{gathered}
\log D_{s t \cdot s}=1,68270+0,01199 A ; \\
D_{s t \cdot s}=48,16 e^{0,02701 \mathrm{~A}}
\end{gathered}
$$

du 7 e mois 15 jours au $46 \mathrm{e}$ mois :

$$
\begin{gathered}
\log D_{\text {st,s }}=1,76218+0,00129 \mathrm{~A} ; \\
D_{\text {st }, \mathrm{s}}=57,97 \mathrm{e}^{0,00297 \mathrm{~A}}
\end{gathered}
$$

Pour la hauteur à la pointe des fesses : du 1 er mols au $6^{\mathrm{e}}$ mois 21 jours :

$$
\begin{gathered}
\log H_{\mathrm{P}}=1,83903+0,02031 \mathrm{~A} ; \\
\mathrm{H}_{\mathrm{P}}=69,03 \mathrm{e}^{0,04677 \mathrm{~A}}
\end{gathered}
$$

du 6 e mois 21 jours au $46 \mathrm{e}$ mois :

$$
\begin{gathered}
\log H_{\mathrm{f}}=1,95918+0,00241 \mathrm{~A} ; \\
H_{\mathrm{f}}=91,03 \mathrm{e}^{\mathbf{0}, 00555 \mathrm{~A}}
\end{gathered}
$$

Le dos forme une légère dépression par rapport au garrot; celle-ci passe de $0,6 \mathrm{~cm}$ à 1 mois à $2,9 \mathrm{~cm}$ à l'âge adulte. La ligne du dos est légèrement inclınée vers l'avant; la différence entre la hauteur aux hanches et la hauteur au dos est sensiblement constante tout au long de la croissance et s'élève en moyenne à $7,5 \mathrm{~cm}$. L'inclinaison du dos, très prononcée dans le jeune âge, s'atténue progressivement au cours de la croissance.

Le bassin présente une inclinaison importante caractérıstıque des races de boucherie ou mixtes. La différence entre la hauteur aux hanches ef la hauteur à la pointe des fesses augmente depuis $12,9 \mathrm{~cm}$ jusqu'à $16,8 \mathrm{~cm}$ à l'âge adulte. Par contre, l'inclinaison moyenne au bassin diminue progressivement au cours de la croissance.

Le rapport entre la distance du sternum au sol et la profondeur de poitrine évolve avec l'âge de 1,62 à 1,08. Le développement de la poitrine en profondeur l'emporte donc sur la crossance en hauteur des sujets.

\section{b) Valeurs moyennes.}

Les différentes hauteurs moyennes des femelles adultes sont respectivement égales à :

- hauteur au garrot ........... $126,3 \mathrm{~cm}$

- hauteur au dos ............ $123,6 \mathrm{~cm}$

- houteur aux hanches ......... $131,8 \mathrm{~cm}$

- hauteur à la pointe des fesses . . $114,9 \mathrm{~cm}$

P. MATHIEU (A. FOCAN, 1959) signale pour le bétail de Luvironza, écotype de taille moindre, $121,4 \mathrm{~cm}$ de hauteur au garrot et $126,5 \mathrm{~cm}$ de hauteur aux hanches. 
Dans une étude du bétall local Nioka, M. MARIEZ (1961) fournit les chiffres ci-après :

$\begin{array}{cc}\text { Hauteur } & \text { Hauteur } \\ \text { au garrot } & \text { aux hanches } \\ \text { en } \mathrm{cm} & \text { en } \mathrm{cm}\end{array}$

$\begin{array}{lrlrr} & & & \overline{1} & \\ \text { Bahema } & 1947 & \ldots \ldots \ldots & 118,5 & 125,5 \\ & 1958 & \ldots \ldots \ldots & 125,5 & 132,8 \\ \text { Alur } & 1947 & \ldots \ldots \ldots & 116,4 & 123,0 \\ & 1958 & \ldots \ldots \ldots & 119,7 & 125,6\end{array}$

Le bétail local Nyamiyaga se distingue parmı les autres écotypes Ankolé par une taille particulièrement élèvée.

\section{c) Dispersion des observations.}

La dispersion des hauteurs au garrot a été étudiée et les renseignements figurent au tableau $n^{\circ} 6$ et au graphique $n^{\circ} 6$.

Les caractéristiques de l'échantillon observé à

\section{Nÿámiyaga sont :}

la moyennex $326,3 \mathrm{~cm}=-\cdots \ldots$.

l'indice de dispersion $\sigma=3,55 \mathrm{~cm}$.

Suivant les résulfafs dês fravaux de divers. auteurs, consignés deras les tableaux no 7,8 et 9 , la hauteur au garrot est en corrélation négative: avec la valeur boucherie des carcasses, tandisi que le rapport tour de poitrine/hauteur au garrot: présente au contraire une corrélation positive: avec la qualité des carcasses.

Au sujet de la production latière, il y a sou-' vent contradiction entre les corrélations calcu-: lées par les divers auteurs sauf, toutefois, pour $\mid \mathrm{a}^{i}$ hauteur au garrot, qui réalıse une corrélation! positive avec la production de lait. Ceci signifie-: rait donc une incompatibilité entre la production laitière et les caractéristıques à la boucherie.

Etant donné que la plupart des auteurs signalent une relation positive entre la hauteur au garrot et la production laitıère et que la hauteur au garrot est en corrélation directe avec la

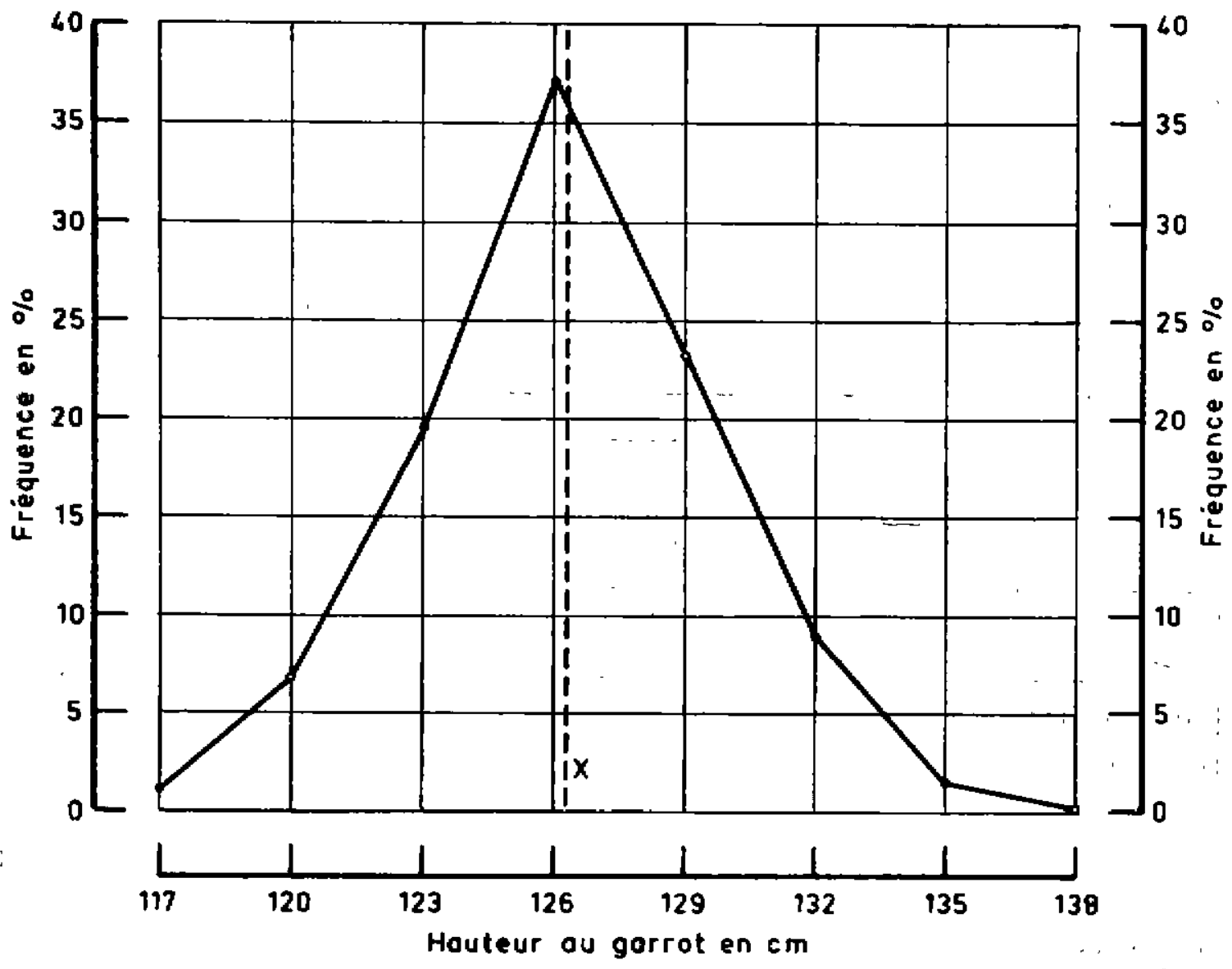

GRAPHIQUE na 6. - Polygone de fréquence des hauteurs au garrol chez les femelles. 
TIBLEAU $n^{0}$ VII

Corrélations entre les meares corporelles of les caractériatiques boucheries chez le même individu

\begin{tabular}{|c|c|c|}
\hline suteurs. & Mensurations & Fendenent en Viande \\
\hline Black, $\underset{(1938)}{\text { Knapp }}$ et Cook & $\begin{array}{l}\text { Hauteur au Earrot } \\
\text { Profondeur de poitrine } \\
\text { Loneueur au corps } \\
\text { Tour de poitrine } \\
\text { Tour de poitrine/hauteur } \\
\text { au Earrot. } \\
\text { Poids Longueur du corps }\end{array}$ & $\begin{array}{l}-0,50 \\
=0,39 \\
=0,49 \\
+0,13 \\
+0,54 \\
+0,54\end{array}$ \\
\hline Cook, Kohli, Dawxon & $\begin{array}{l}\text { Hauteus au garrot } \\
\text { Hauteur au niveau de la poitrine } \\
\text { Longueur du corps } \\
\text { Largeur aux épaules } \\
\text { Tour de poitrine }\end{array}$ & $\begin{array}{l}-0,20 \\
-0,18 \\
-0,20 \\
+0,08 \\
-0,02\end{array}$ \\
\hline $\begin{array}{c}\text { Yao, Dawsong Cook } \\
(1953)\end{array}$ & $\begin{array}{l}\text { Heuteur de garrot } \\
\text { Tour de poitrine }\end{array}$ & $\begin{array}{l}+0,01 \\
+0,42\end{array}$ \\
\hline
\end{tabular}

TABLEAU NO VIII

Corrélations génétiques estimées par deux méthodes a et b entre les mensurations prises à 3 âges et la production laztrère.

(Blackmore et al, 1958).

\begin{tabular}{|l|c|c|c|c|c|c|}
\hline \multirow{4}{*}{} & \multicolumn{2}{|c|}{ 6 mois. } & \multicolumn{2}{c|}{1 an. } & \multicolumn{2}{c|}{2 ans. } \\
\cline { 2 - 7 } & $\mathrm{a}$ & $\mathrm{b}$ & $\mathrm{a}$ & $\mathrm{b}$ & & \\
\cline { 2 - 7 } Hauteur au garrot & $+0,17$ & $+0,34$ & $+0,21$ & $+0,32$ & $+0,23$ & $+0,34$ \\
Profondeur de poitrine & $-0,30$ & $+0,35$ & $-0,22$ & $-0,03$ & $-0,23$ & $+0,14$ \\
Longueur du corps & $-0,50$ & $+0,25$ & $-0,35$ & $+0,32$ & $-0,12$ & $+0,35$ \\
Tour de poitrine & $-0,51$ & $-0,05$ & $-0,66$ & $-0,13$ & $-0,34$ & $+0,10$ \\
Tour d'abdomen & $-0,30$ & $+0,68$ & $-0,22$ & $+0,46$ & $-0,13$ & $+0,37$ \\
Poids & $+0,01$ & $+0,10$ & $+0,00$ & $+0,17$ & $-0,02$ & $+n, 14$ \\
\hline
\end{tabular}

RABLEAO NO II

Corrélations génétiques et phénotjpiques entro la production laitièreet les meneuratione

(P.Aurtol ot al, 1961).

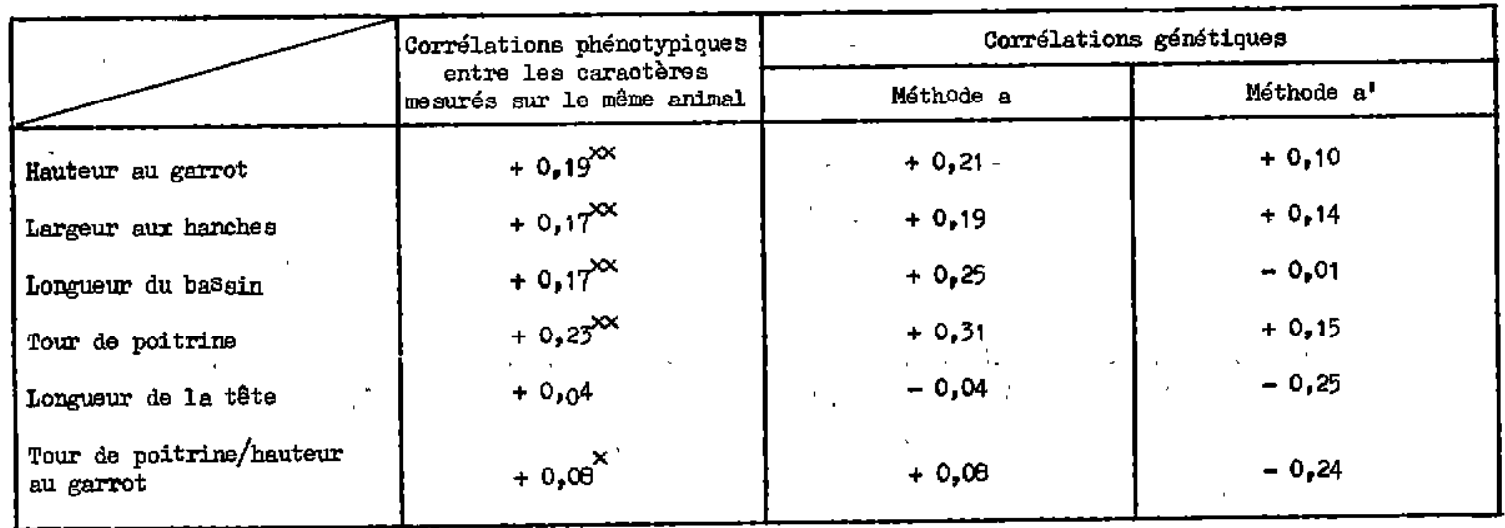


longueur du corps, la sélection tend à retenur les animaux possédant une hauteur satisfaisante ; c'est-à-dire entre 124 et $130 \mathrm{~cm}$.

\section{d) Relations entre les observations.}

\section{Animaux en croissance.}

Croissance relative du poids vif et de la hauteur au garrot (graphique $n^{0} 2$ ).

Le coefficient de corrélation entre le poids vif et la hauteur au garrot s'élève à 0,97, valeur hautement significative.

La relation mathématique entre les deux variables s'exprime par la formule: ou

$\log$ Poids $=4,06497 \log H_{g}-6,02230$

$$
\text { Poids }=0,000000950 \mathrm{Hg}_{\mathrm{g}}{ }^{4,06}
$$

J. PAGOT et col. obtiennent une relation semblable sur le bétail N'Dama où

$$
\text { Polds }=0,000001031 \mathrm{Hg}_{\mathrm{g}}^{3,57}
$$

\section{Femelles adultes.}

Corrélation entre la longueur du corps ef la hauteur au garrot (voir ci-dessus).

\section{Les MALES.}

Les mâles sont en moyenne beaucoup plus hauts que les femelles avec les dimensions moyennes suivantes:

$$
\text { Taurecux Mâles }
$$$$
\text { de sélection castrés }
$$

Age moyen ......... 67 mois 45 mois

Hauteur au garrot. ..... $132,0 \mathrm{~cm} \quad 128,8 \mathrm{~cm}$ Hauteur au dos ........ $129,3 \mathrm{~cm} \quad 127,0 \mathrm{~cm}$ Houteur aux hanches.... $138,0 \mathrm{~cm} \quad 135,6 \mathrm{~cm}$ Hauteur à la pointe des

fesses ............ $115,3 \mathrm{~cm} \quad 116,6 \mathrm{~cm}$

Les équations donnont les hauteurs en centimètres en fonction de l'age en mois sont :

Pour la hauteur au garrot:

du 1 er mois au 6 e mois :

$$
\begin{gathered}
\log H_{\mathrm{g}}=1,88545+0,02144 \mathrm{~A} ; \\
H_{\mathrm{g}}=76,82 \mathrm{e}^{0,04937 \mathrm{~A}}
\end{gathered}
$$

Au-delà du 12e mois chez les taureaux :

$$
\begin{gathered}
\log H_{g}=2,03595+0,00236 \mathrm{~A} ; \\
H_{g}=108,6 \mathrm{e}^{\mathbf{0 . 0 0 5 4 3 \mathrm { A }}}
\end{gathered}
$$

Au-delà du $12^{\mathrm{e}}$ mois chez les boufs :

$$
\begin{gathered}
\log H_{g}=2,00426+0,00242 \mathrm{~A}: \\
H_{g}=101,0 \mathrm{e}^{0,00557 \mathrm{~A}} .
\end{gathered}
$$

Pour la haufeur au dos:

du 1 er mois au 6 e mois :

$$
\begin{gathered}
\log H_{d}=1,87671+0,02185 \mathrm{~A} ; \\
H_{d}=75,28 \mathrm{e}^{\mathbf{0}, 05031 \mathrm{~A}}
\end{gathered}
$$

Au-delà du $12^{e}$ mois chez les taureaux :

$$
\begin{gathered}
\log H_{d}=2,03199+0,00222 \mathrm{~A} \text {; } \\
H_{d}=107,6 \mathrm{e}^{0,00511 \mathrm{~A}}
\end{gathered}
$$

Au-delà du $12^{\mathrm{e}}$ mois chez les bœufs :

$$
\begin{gathered}
\log H_{d}=2,00552+0,00225 \mathrm{~A}: \\
H_{d}=101,3 \mathrm{e}^{0,00518 \mathrm{~A}}
\end{gathered}
$$

Pour lo hauteur aux hanches:

du 1 er mals au 6 e mois :

$$
\begin{gathered}
\log H_{h}=1,93678+0,01612 \mathrm{~A} ; \\
H_{h}=86,45 \mathrm{e}^{0,03712 \mathrm{~A}}
\end{gathered}
$$

Au-delà du $12^{e}$ mois chez les taureaux :

$$
\begin{gathered}
\log H_{h}=2,06110+0,00225 \mathrm{~A} \text {; } \\
H_{h}=115,1 e^{0,00518 \mathrm{~A}}
\end{gathered}
$$

Au-delà du $12^{\mathrm{e}}$ mois chez les bœufs :

$$
\begin{gathered}
\log H_{h}=2,03407+0,00227 \mathrm{~A} ; \\
H_{\mathbf{h}}=108,1 \mathrm{e}^{\mathbf{0 . 0 0 5 2 3} \mathrm{A}}
\end{gathered}
$$

Pour la hauteur à la poinfe des fesses : du 1 er mois au $6^{e}$ mols :

$$
\begin{gathered}
\log H_{P}=1,84177+0,02041 \mathrm{~A} ; \\
H_{\mathrm{I}}=69,47 \mathrm{e}^{0,4700 \mathrm{~A}} .
\end{gathered}
$$

Au-delà du $12^{\mathrm{e}}$ mois chez les taúreaux :

$$
\begin{gathered}
\log H_{\mathrm{r}}=1.99739+0.00198 \mathrm{~A} ; \\
H_{\mathrm{P}}=99.40 \mathrm{e}^{0.00450 \mathrm{~A}}
\end{gathered}
$$

Au-delà du $12 \mathrm{e}$ mois chez les bœufs :

$\log H_{P}=1,98408+0,00194 \mathrm{~A}$;

$$
H_{t}=96,40 e^{0.00447 A}
$$

Pour la distance du sternum au sol: du 1 er mois au be mois :

$$
\begin{gathered}
\log D_{\text {st } \cdot \mathrm{s}}=1,68472+0,01356 \mathrm{~A} ; \\
D_{\text {st.s }}=48,39 \mathrm{e}^{\mathbf{0 , 0 3 1 2 2} \mathrm{A}}
\end{gathered}
$$

Au-delà du $12^{\mathrm{e}}$ mois chez les taureaux :

$$
\begin{gathered}
\log D_{\text {st } \cdot \mathrm{s}}=1,79278+0,00116 \mathrm{~A} ; \\
D_{\text {st } \cdot \mathrm{s}}=62,06 \mathrm{e}^{\mathbf{0 , 0 0 2 6 7 \mathrm { A }}}
\end{gathered}
$$

Au-delà du $12^{\mathrm{e}}$ mois chez les boufs :

$$
\begin{gathered}
\log D_{\text {st } \cdot \mathrm{s}}=1,76390+0,00151 \mathrm{~A}: \\
D_{\text {st } \cdot \mathrm{s}}=58,06 \mathrm{e}^{\mathbf{0 . 0 0 3 4}}=5
\end{gathered}
$$


Le bassin des mâles est beaucoup plüs incliné que celul des femelles. Le choix des géniteurs, en recherchant une croupe large et harmonieusement développée, augmente chez ces derniers l'inclinaison de la croupe.

Le rapport entre la distance du sternum aú sól et la profondeur de poitrıne est à peu près égal chez les femelles et les mâles castrés, mais nettement moins élevé chez les taureaux, car les reproducteurs mâles sont sélectionnés tout particulıèrement suivant la profondeur de l'avantmain.

\section{IV. - CONFORMATION DE LA POITRINE}

Le bétail Ankolé possède une bonne profondeur de poitrıne mais elle manque généralement de largeur. En choisissant des taureaux particulièrement avantagés dans l'avant-main, le sélectionneur tâche d'augmenter ces caractéristiques ef de donner au bétail une bonne ampleur de poitrine.

\section{A. Les mensurations.}

Pour estimer le développement de la cage thoracique, on a procédé aux mensurations suivantes :

- la largeur de poitrine : mesurée en arrière des épaules:

- la profondeur de poitrıne : prise derrière la pointe du coude depuis le sternum jusqu'au dos ;

- le périmètre thoracique: pris à l'aide du ruban immédıatement en arrière des épaules.

\section{B. LeS fEMELLES.}

Les excellentes femelles extériorisent une grande profondeur de poitrine accompagnée d'un périmètre thoracique dépassant $180 \mathrm{~cm}$.

\section{a) Développement de la poitrine.}

La croissance de la poitrine en largeur s'arrête entre 2 ans et demi et 3 ans tandis que le développement en profondeur dure becucoup plus longtemps, c'est-à-dire jusqu'à 3 ans ef demi à 4 ans en moyenne.

A Mulungu (COMPËRE, R., 1960), la cage thoracique du demi-sang Brun-Suisse se termine vers 4 ans et demi.

J. PAGOT et col. (1959) signalent un arrêt assez précoce du développement de la cage tho- racique chez le bétall N'Dam'a. AInsi, l'augimenta' tion du périmètre thoracique de ce bétail aú delà de la $3 e$ année est cheź le mâle foniction dè l'embonpoint et chez la femelle fonction à la fois du stade de gestation et de l'état d'embónipoint.

Les équations donnant les dimensions de la poitrine.: largeur de la poitrine « $|p\rangle$, profondeur de la poitrine «PP» et pérımètre thoracique $\ll P_{\text {th }}$ » exprimées en centımètres en fonction derl'âge «A $A$ en mois, sont:

Pour lo largeur de poitrine:

du' 1 er mols au be mols :

$$
\begin{gathered}
\log I p=1,211.72+0,023.41 \mathrm{~A}: \\
I P=16,28 \mathrm{e}^{0,05994 \mathrm{~A}}
\end{gathered}
$$

du $6^{\mathrm{e}}$ mois au $36^{\mathrm{e}}$ mois :

$$
\begin{gathered}
\log I p=1,324.92+0,004.58 \mathrm{~A} ; \\
I p=21,13 \mathrm{e}^{0.01055 \mathrm{~A}}
\end{gathered}
$$

Pour la profondeur de poitrine :

du 1 er mols au 5 e mols 28 jours :

$$
\begin{gathered}
\log P_{p}=1,45303+0,03111 A ; \\
P_{p}=28,38 e^{\mathbf{0 . 0 7 1 6 3 A}} ;
\end{gathered}
$$

Du $5 \mathrm{e}$ mois 28 jours au $46 \mathrm{e}$ mois :

$$
\begin{gathered}
\log P_{p}=1,61390+0,00403 A^{\prime} ; \\
P_{p}=41,11 e^{0,00928 A}
\end{gathered}
$$

Pour le périmètre thoracique :

du 1 er mois au 6 e mois :

$$
\begin{gathered}
\log P_{\text {th }}=1,95983+0,02657 \mathrm{~A} ; \\
P_{\text {th }}=-91,17 \mathrm{e}^{0,06118 \mathrm{~A}}
\end{gathered}
$$

du $6^{\mathrm{e}}$ mois au $46^{\mathrm{e}}$ mois :

$$
\begin{gathered}
\log P_{\text {th }}=2,09865+0,00340 \mathrm{~A} ; \\
P_{\text {th }}=125,5 \mathrm{e}^{0.00783 \mathrm{~A}}
\end{gathered}
$$

La cage thoracique se développe légèrement plus en profondeur qu'en largeur ce qui a pour effet d'allonger sa section jusqu'à un rapport largeur-profondeur égal à 0,5 .

\section{b) Valeurs moyennes.}

Les dimensions moyennes de la cage thoracique du lacal Nyamiyaga sont les suivantes:

largeur de poitrine ...... $31,9 \mathrm{~cm}$

profondeur de poitrıne.... $61,7 \mathrm{~cm}$

périmètre thoracique ...... $176,0 \mathrm{~cm}$

Pour le bétail de type Ankolé de Luvironza, P. MATHIEU (A. FOCAN, 1959) signale $26,2 \mathrm{~cm}$ 
de largeur de poitrıne, $58,6 \mathrm{~cm}$ de profondeur de poitrine et $158,9 \mathrm{~cm}$ de périmètre thoracique.

M. MARICZ (1961) publie pour le bétail de I'Iturı les chiffres suivants:

\begin{tabular}{|c|c|c|c|}
\hline. & & $\begin{array}{l}\text { Profondeur } \\
\text { de poitrine } \\
\text { en } \mathrm{cm}\end{array}$ & $\begin{array}{c}\text { Périmètre } \\
\text { thoradique } \\
\text { en } € m\end{array}$ \\
\hline & & $\overrightarrow{587}$ & 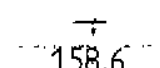 \\
\hline aher & $\begin{array}{l}1947 \\
1958\end{array}$ & 60,8 & $\begin{array}{l}150,0 \\
163,4\end{array}$ \\
\hline Alur & $\begin{array}{l}1947 \\
1958\end{array}$ & $\begin{array}{l}61,8 \\
63,6\end{array}$ & $\begin{array}{l}159: 2 \\
168.8\end{array}$ \\
\hline
\end{tabular}

Comparé aux autres-5ous-types, le local Nyamlyaga extériorise donc une cage thoracique de bonne ampleur.

\section{c) Dispersion des observations.}

La dispersion des périmètres thoraciques des femelles adultes a été étudiée (tableau no 10 et graphique no 7).

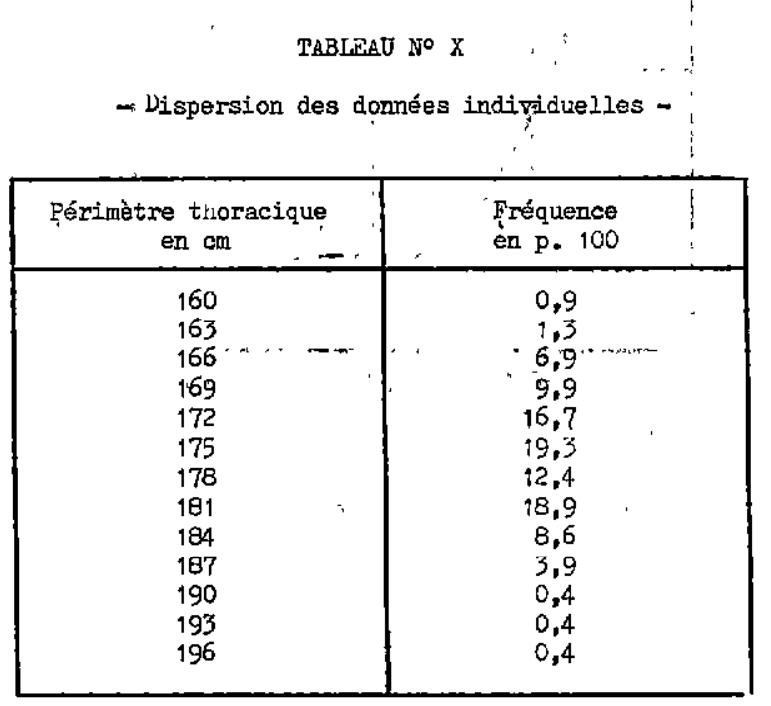

Les caractéristiques de l'échantillon observé sont :

Valeur moyenne $\bar{X}=176,0 \mathrm{~cm}$.

Indice de dispersion $\sigma=6,18 \mathrm{~cm}$.

Certaines mensurations peuvent apporter des indications intéressantes quant aux aptitudes bouchères des individus. Le tour de poitrine et surtout le rapport tour de poitriné/hauteur au garrot présentent, suivant les auteurs, une corrélation positive avec la qualité des carcasses.
Suivant Lush (1952) un rendement élevé est associé da un tour de poitrine important, à un tour d'abdomen et à une profondeur de poitrine faibles. II signale une corrélation de $+0,56$ entre le rapport tour de poitringe/hauteur au garrot et l'état d'engraissement de l'anımal.

P. AURIOL let col (1961) trouvent une corréIation hautement significative entre la production laitière ef le-tâu de poitrine, et une corrélation significative entre la production laitière et le rapport tour de poitrine/hauteur au garrot.

- A la Jumière de ces quelques informations, laugmentation du tour Ide poitrıne du bétail lócal doit être poursoivie d'autant plus que cette dimensıon"est en corrélation étroite avec le poids vif. On recherchera les femelles possédant un périmètre thoracique supérieur à $180 \mathrm{~cm}$ ce qui représente $32,6 \%$ de la population.

\section{d) Relations entre les observations.}

\section{Animoux en croissance.}

Croissance relative du poids et du périmètre thoracique (graphique $n^{\circ} 2$ ).

On obtient lo relation suivante :

ov

$$
\log \text { Poids }=3,27633 \log P_{t h}^{-4,81441}
$$

$$
\text { Poids }=0,0000153 \mathrm{P}_{\mathrm{th}^{3,28}}
$$

La relation obtenue par 1. PAGOT pour le bétail N'Dama est représentée par l'équation :

$$
\text { Poids }=0,000374 \mathrm{P}_{\mathbf{t h}}{ }^{2,62}
$$

\section{Femelles adultes.}

Corrélation entre le poids vif et le périmètre thoracique.

Il existe une corrélation de $+0,70$ hautement significative entre le poids vif et le périmètre thoracique.

Corrélation entre la hauteur au garrot et la profondeur de poitrine.

Etant donné qu'il est aisé d'allier chez un même animal l'augmentation de la longueur du corps et de la hauteur au garrot, il est utile de connaître ensuite la relation statıstique qui unit la hauteur au garrot d'une part et la profondeur en poitrine d'autre part.

Le coefficient de corrélation qui chiffre le lien entre ces deux grandeurs chez un même individu, s'élève à $+0,49$, valeur hautement signi1 ficative. Ceci signifie que les animaux très hauts 


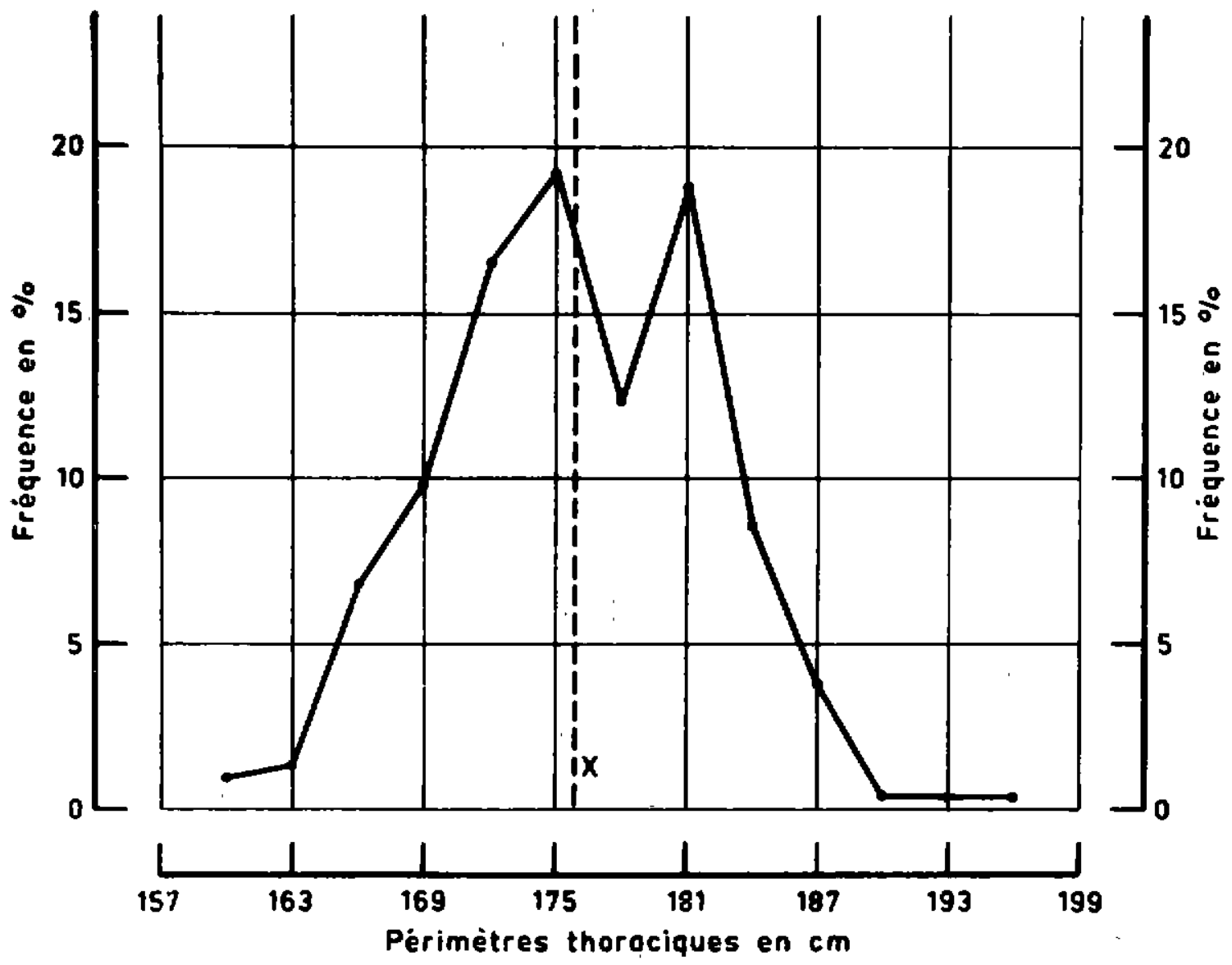

GRAPHIQUE n० 7. - Polygone de fréquence des périmètres thoraciques chez les femelles.

possèdent également une bonne profondeur de poitrine.

\section{Les males.}

Les mâles offrent une poitrine beaucoup plus développée que celle des femelles : surtout les géniteurs sélectionnés qui ont été choisis principalement suivant les proportions de l'avantmain.

Les dimensions moyennes sont les suivantes : Mâles

Taureaux castrés

$\begin{array}{llcr} & \text { - } & \text { - } \\ \text { Age moyen .......... } & 67 \text { mois } & 45 \text { mois } \\ & & 21 \text { jours } \\ \text { Largeur de poltrine...... } & 43,7 \mathrm{~cm} & 31,8 \mathrm{~cm} \\ \text { Profondeur de poitrine ... } & 68,0 \mathrm{~cm} & 61,0 \mathrm{~cm} \\ \text { Périmètre thoracique .... } & 206,3 \mathrm{~cm} & 174,8 \mathrm{~cm}\end{array}$


Les équations donnant les dimensions de la poitrine en centimètres en fonction de l'âge en mois, sont :

Pour la largeur de poitrine : du 1 er mois au 6 e mois :

$$
\begin{gathered}
\log 1 p=1,23263+0,02314 \mathrm{~A} ; \\
I p=17,09 \mathrm{e}^{0,05928 \mathrm{~A}}
\end{gathered}
$$

Au-delà du $12^{\mathrm{e}}$ mois chez les taurequx :

$$
\begin{gathered}
\log I P=1,35113+0,00549 A ; \\
I P=22,45 e^{0,02264 A}
\end{gathered}
$$

Au-delà du $12^{\mathrm{e}}$ mois chez les boufs :

$\log I P=1,34225+0,00367 \mathrm{~A}$;

$$
I p=21,89 \mathrm{e}^{0,00845 \mathrm{~A}}
$$

Pour la profondeur de poitrine :

du 1 er mors au $6^{e}$ mols :

$$
\begin{gathered}
\log P P=1,45628+0,03286 \mathrm{~A} ; \\
P p=28,60 \mathrm{e}^{0,07566 \mathrm{~A}}
\end{gathered}
$$

Au-delà du $12^{\mathrm{e}}$ mois chez les taureaux :

$$
\begin{gathered}
\log P_{P}=1,67122+0,00370 \mathrm{~A} ; \\
P p=46,90 \mathrm{e}^{0.00852 \mathrm{~A}}
\end{gathered}
$$

Au-delà du $12^{e}$ mols chez les boufs :

$$
\begin{gathered}
\log P_{P}=1,65295+0,00314 A ; \\
P p=44,97 e^{0,00723 A}
\end{gathered}
$$

Pour le périmètre thorocique :

du 1 er mois au 6 e mois :

$$
\begin{gathered}
\log P_{\text {th }}=1,95806+0,03058 \mathrm{~A} ; \\
P_{\text {th }}=90,79 \mathrm{e}^{\mathbf{0}, 07041 \mathrm{~A}}
\end{gathered}
$$

Au-delà du $12^{\mathbf{e}}$ mois chez les taureaux :

$\log P_{\text {th }}=2,13979+0,00377 \mathrm{~A}$;

$$
P_{\text {th }}=137,9 \mathrm{e}^{0,00868 \mathrm{~A}}
$$

Au-delà du $12^{\mathrm{e}}$ mois chez les bœufs :

$$
\begin{gathered}
\log P_{\text {th }}=2.12624+0,00277 \mathrm{~A} \text {; } \\
P_{\text {th }}=133,7 \mathrm{e}^{\mathbf{0 , 0 0 6 3 8} \mathrm{A}}
\end{gathered}
$$

La forme de la cage thoracique des castrés est identique à celle des femelles tandis qu'elle est moins elliptıque chez les reproducteurs.

\section{V. - CONFORMATION DE LA RÉGION LOMBAIRE}

\section{A. Mensurations.}

Seule la largeur du rein a été abservée : c'est-àdire la distance entre les apophyses transverses des vertèbres lombares.

\section{B. Les feMELLES.}

La largeur du rein qui résulte plus souvent du développement des muscles que de l'élongation des apophyses vertébrales est une beauté absolve. Un rein large harmonieusement attaché aux autres parties du corps est une caractéristique à rechercher.

\section{a) Développement du rein.}

Le rein a pratiquement terminé son développement entre 4 ans et 4 ans et demi. Les équations donnant la largeur du rein « $I_{\mathbf{r}}$ » exprimée en centimètres, en fonction de l'âge « $A$ 》 en mois, sont :

Du $1^{\text {er }}$ mols au 6 e mols 18 jours :

$$
\begin{gathered}
\log I_{\mathrm{r}}=1,13004+0,03007 \mathrm{~A} ; \\
I_{\mathrm{r}}=13,49 \mathrm{e}^{0,06294 \mathrm{~A}}
\end{gathered}
$$

du $6^{\mathrm{e}}$ mois 18 jours au $46^{\mathrm{e}}$ mois :

$$
\begin{gathered}
\log I_{r}=1,29607+0,00493 A \text {; } \\
I_{r}=19,77 e^{0.01135 A}
\end{gathered}
$$

b) Valeur moyenne.

La largeur moyenne du rein est de $33,7 \mathrm{~cm}$. P. MATHIEU (A. FOCAN, 1959) signale une valeur moyenne de $31,4 \mathrm{~cm}$ pour le local Luvironza.

\section{c) Dispersion des observations.}

La dispersion des largeurs du rein figure au tableau no 11 et. au graphique n० 8 . Les caractéristıques de l'échantillon observé à Nyamiyaga sont :

Valeur moyenne $\bar{X}=33,7 \mathrm{~cm}$.

Indice de dispersion $\sigma=2,06 \mathrm{~cm}$.

TABLGAO No II

DLepersion des données Indfviduelles

\begin{tabular}{|c|c|}
\hline Largeur du rein en cm & $\begin{array}{r}\text { Fréquence } \\
\text { en p.100 }\end{array}$ \\
\hline 28,5 & 2,7 \\
30,5 & 10,3 \\
32,5 & 31,4 \\
34,5 & 37,8 \\
36,5 & 16,2 \\
38,5 & 1,1 \\
40,5 & 0,5 \\
\hline
\end{tabular}


Un rein d'une largeur supérieure à $34 \mathrm{~cm}$ est recherché, ce qui représente approximativement $55,6 \%$ des valeurs observées.

\section{Les maLes.}

Les boufs ont une largeur du rein égale à celle des femelles tandis que les géniteurs choisis possèdent un rein nettement plus musclé. Les valeurs moyennes sont :

Age moyen du rein

Bœufs ........ 45 mois 21 jours $31,5 \mathrm{~cm}$

Tqureaux..... 67,0 mols $\ldots \ldots, 36,7 \mathrm{~cm}$
Les équations donnant la largeur du rein exprımée en centimètres en fonction de l'âge en mois, sont :

du 1 er au $6 \mathbf{e}$ mois :

$$
\begin{gathered}
\log I_{r}=1,12551+0,03387 \mathrm{~A} ; \\
I_{r}=1,3,35 \mathrm{e}^{\mathbf{0 . 0 7 7 9 9 A}}
\end{gathered}
$$

Au-delà du $12^{\mathrm{e}}$ mois chez les taureaux,

$$
\log I_{\mathbf{r}}=1,33805+0,00528 \mathrm{~A} \text {; }
$$$$
\mathrm{I}_{\mathrm{r}}=21,78 \mathrm{e}^{0,01216 \mathrm{~A}}
$$

Au-delà du $12^{\mathrm{e}}$ mois chez les bcufs :

$$
\begin{gathered}
\log I_{r}=1,31176+0,00443 A: \\
I_{r}=20,50 e^{0,01020 A}
\end{gathered}
$$

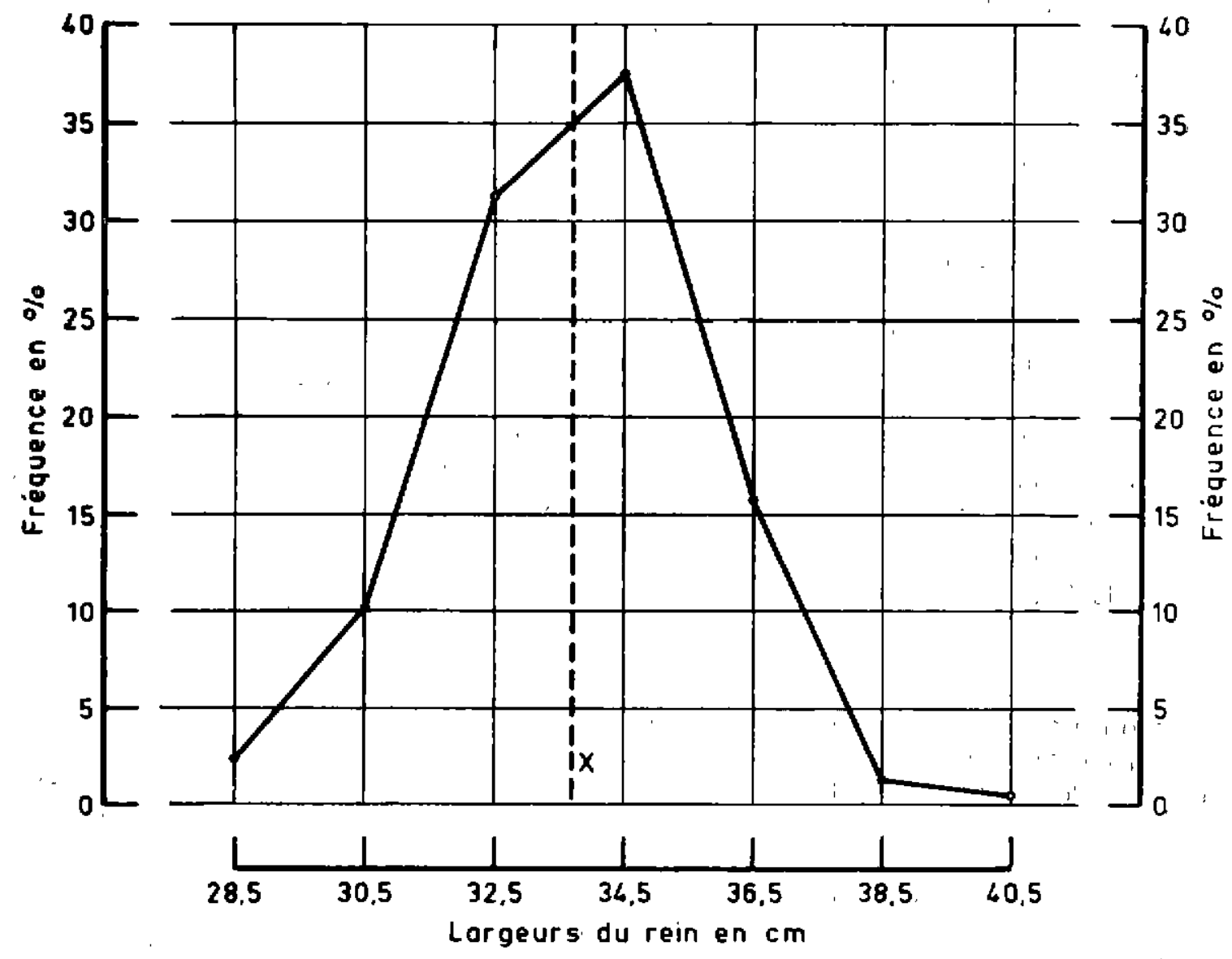

GRAPHIQUE no 8. - Polygone de fréquence des largeurș du rein chez les femelles. 


\section{VI. - CONFORMATION DU BASSIN}

\section{A. Mensurations.}

Les mensurations suivantes ont été réalisées :

- largeur aux hanches : entre les pointes extérieures des hanches :

- largeur aux articulations coxo-fémorales : de la tête d'un des fémurs à l'autre ;

- largeur aux pointes des fesses : entre les bords externes des deux tubérosités ischiatiques ;

- longueur du bassin : du bord extérieur de la hanche jusqu'à la pointe des fesses.

\section{B. LeS FEMELLES.}

Cette région constitue une qualité absolve chez les femelles; un bassin ample conditionne l'espace nécessaire à une mamelle bien développée et à une filière pelvienne suffisante pour livrer passage à des veaux de bon format.

\section{a) Développement du bassin.}

Le développement de la largeur aux hanches se termine en dernier lieu, c'est-à-dire entre 4 ans et demi et 5 ans, tandis que la largeur aux articulations coxo-fémorales ef la largeur à la pointe des fesses sont déjà définitıvement formées entre 3 ans ef 3 ans et dems. La longueur du bassin poursuit sa croissance jusqu'à 3 ans et demi à 4 ans.

A Mulungu (R. COMPERE, 1960), le bassin des demi-sang Bruns-Suisses poursuit son développement jusqu'ò 5 ans.

La forme du bassin évolue avec l'âge de la façon suivante (graphıque no 9) :

- légèrement hexagonale jusqu'à 6 mois,

- carrée à la base de 7 mols à 1 an ef demi,

- de plus en pius trapézoïdale jusqu'à 4 ans et demi à 5 ans, époque à laquelle le bassın a acquis sa forme définitive.

Les équations donnant les dimensions du bassin : largeur aux hanches $\left.«\right|_{h}$ », largeur aux articulations coxo-fémorales $\left.\left.«\right|_{c \cdot t}\right\rangle_{1}$ largeur d la pointe des fesses $\left\langle\left.\right|_{p} \cdot p\right.$ » et longueur du bas$\sin \left\langle L_{\mathbf{b}}\right.$ » en centimètres, en fonction de l'âge «A» en mois, sont :

Pour la largeur aux hanches :

du 1 er mois au 6 e mois 8 jours :

$$
\begin{gathered}
\log l_{\mathbf{h}}=1,22834+0,03614 \mathrm{~A} ; \\
l_{\mathbf{h}}=16,92 \mathrm{e}^{\mathbf{0 . 0 3 3 2 2 A}}
\end{gathered}
$$

du $6^{\mathrm{e}}$ mois 6 jours au, $46^{\mathrm{e}}$ mois :

$$
\begin{gathered}
\log I_{h}=1,42467+0,00479 A ; \\
I_{h}=26,59 e^{0,01103 A}
\end{gathered}
$$

Pour la lorgeur aux articulations coxo-fémorales: du $1 \mathrm{er}$ mois au $6^{\mathrm{e}}$ mois 14 jours :

$$
\begin{gathered}
\log l_{\mathbf{c} \cdot \mathbf{f}}=1,28501+0,02594 \mathrm{~A} ; \\
I_{\mathbf{c} \cdot \mathfrak{P}}=19,28 \mathrm{e}^{\mathbf{0 . 0 5 9 7 3 A}}
\end{gathered}
$$

du $6^{\mathrm{e}}$ mois 14 jours au $36^{\mathrm{e}}$ mois :

$$
\begin{gathered}
\log I_{\mathbf{c} \cdot \mathrm{f}}=1,43134+0,00327 \mathrm{~A}: \\
I_{\mathbf{c} \cdot \mathrm{P}}=27,00 \mathrm{e}^{\mathbf{0}, 00753 \mathrm{~A}}
\end{gathered}
$$

Pour la largeur à la pointe des fesses:

du 1 er mois au 5 e mois 3 jours :

$$
\begin{gathered}
\log l_{\mathbf{p} \cdot \mathbf{q}}=1,08848+0,02089 \mathrm{~A} ; \\
l_{\mathbf{p} \cdot \mathbf{f}}=12,26 \mathrm{e}^{0,04810 \mathrm{~A}}
\end{gathered}
$$

du 5 e mois 3 jours au $36^{e}$ mois :

$$
\begin{gathered}
\log I_{p \cdot f}=1,17378+0,00421 A ; \\
I_{p \cdot f}=14,92 \mathrm{e}^{0,0086 \theta A}
\end{gathered}
$$

Pour lo longueur du bassin :

du 1 er mois au 5 e mois 8 jours :

$$
\begin{gathered}
\log L_{b}=1,35880+0,02954 A ; \\
L_{b}=22,85 e^{0,0682 A}
\end{gathered}
$$

du $5^{\mathrm{e}}$ mols 8 jours au $46^{\mathrm{e}}$ mois :

$$
\begin{gathered}
\log L_{b}=1,49272+0,00416 \mathrm{~A} ; \\
L_{\mathbf{b}}=31,10 \mathrm{e}^{\mathbf{0 , 0 0 9 5 8}}
\end{gathered}
$$

A 40 mois, époque moyenne des vêlages, la forme du bassin esł très satisfaisante pour permettre un passage aisé du produit. Ceci reporte l'âge moyen des saillies vers 2 ans et demi, ce qui correspond au format moyen du bassin suivant :

- largeur aux hanches : $37,0 \mathrm{~cm}$,

- largeur aux articulations coxo-fémorales:

$33,8 \mathrm{~cm}$,

- largeur à la pointe des fesses: $20,0 \mathrm{~cm}$,

- longueur du bassin : $41,5 \mathrm{~cm}$.

Ces données servent actuellement de critère pour fixer l'époque de la mise au taureau.

En outre, le rapport langueur/largeur varie au cours de la croissance : allongé durantle jeune

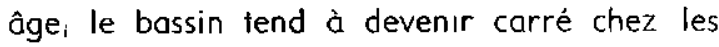
adultes.

\section{b) Valeurs moyennes.}

Les dimensions moyennes du bassin des femelles adultes sont les suivantes:

- largeur aux hanches : $44,0 \mathrm{~cm}$, 


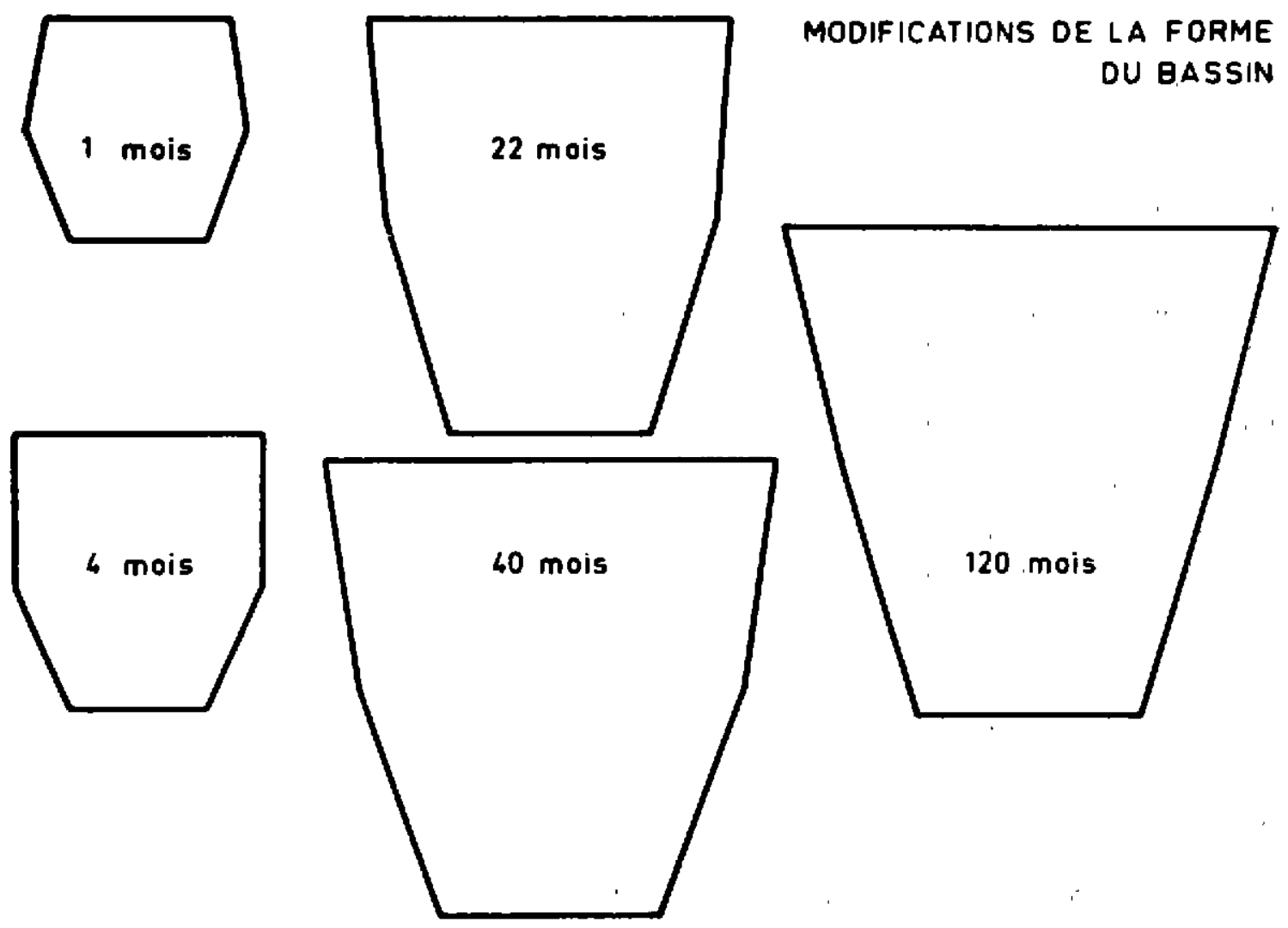

GRAPHIQUE n 9. - Modifications de la forme du bassin chez les femelles.

- largeur aux articulations coxo-fémorales: $37,1 \mathrm{~cm}$,

- largeur à la pointe des fesses : 22,2 cm,

- longueur du bassin : $46,8 \mathrm{~cm}$.

P. MATHIEU (A. FOCAN, 1959) signale pour le bétail local Luvironza $42,6 \mathrm{~cm}$ de largeur aux hanches, $36,0 \mathrm{~cm}$ de largeur aux articulations coxo-fémorales et $42,6 \mathrm{~cm}$ de longueur du bassın.

M. MARICZ fournit pour le lacal Nioka, les chiffres suivants :

$\begin{array}{cc}\text { Longueur } & \text { Largeur } \\ \text { du bassin aux hanches } \\ \text { en } \mathrm{cm} & \text { en } \mathrm{cm}\end{array}$

$\begin{array}{lllll} & & & & \\ \text { Bahema } & 1947 & \ldots \ldots \ldots & 41,9 & 40,0 \\ \text { Alur } & 1958 & \ldots \ldots \ldots & 44,5 & 42,0 \\ & 1947 & \ldots \ldots \ldots & 43,0 & 40,4 \\ & 1958 & \ldots \ldots \ldots & 45,2 & 42,5\end{array}$

Comparé aux sous-types des autres, régions, le lacal Nyamiyaga possède un bassin d'un format particulièrement développé.

\section{c) Dispersion des observations.}

La dispersion des largeurs aux hanches a été étudiée (tableau no 12 et graphique no 10 ).

Les caractéristiques de l'échantillon sont :

Valeur moyenne $\bar{X}=44,0 \mathrm{~cm}$.

Indice de dispersion $\sigma=1,87 \mathrm{~cm}$.

P. AURIOL et col. (1961) calculent une corrélation de $+0,17$ hautement significative entre la largeur aux hanches ef la production laitière, ce qui fait supposer une relation entre l'ampleur du bassin et la valeur laitière de la femelle.

A Nyamiyaga, la sélection retient particulièrement les vaches dont la largeur aux hanches dépasse $45 \mathrm{~cm}$, ce : qui représente 40,7 p. 100 de la population étudiée. 
TABLEAU No XII

Dispersion des données individuelles

\begin{tabular}{|c|c|}
\hline $\begin{array}{c}\text { Largeur aur hanches } \\
\text { en cm }\end{array}$ & $\begin{array}{r}\text { Fréquence } \\
\text { en p.100 }\end{array}$ \\
\hline 39 & 0,5 \\
40 & 1,6 \\
41 & 6,0 \\
42 & 14,1 \\
43 & 15,2 \\
44 & 21,7 \\
45 & 21,2 \\
46 & 9,2 \\
47 & 7,6 \\
48 & 1,1 \\
49 & 1,6 \\
\hline
\end{tabular}

d) Relations entre les observations.

Femelles odultes.

Corrélation entre la largeur aux hanches ef la longueur du corps :

Le coefficient de corrélation calculé entre la largeur aux hanches et la longueur du corps chez un même individu s'élève à $+0,399 \times x>$ 0,321 (valeur limite au seuil $P=0,001$ ). Ceci signifie qu'il existe une relation positive et hautement significative entre ces deux grandeurs. Actuellement, eile est exploitée très activement par la sélection.

Corrélation entre la hauteur au garrot ef la largeur aux hanches.

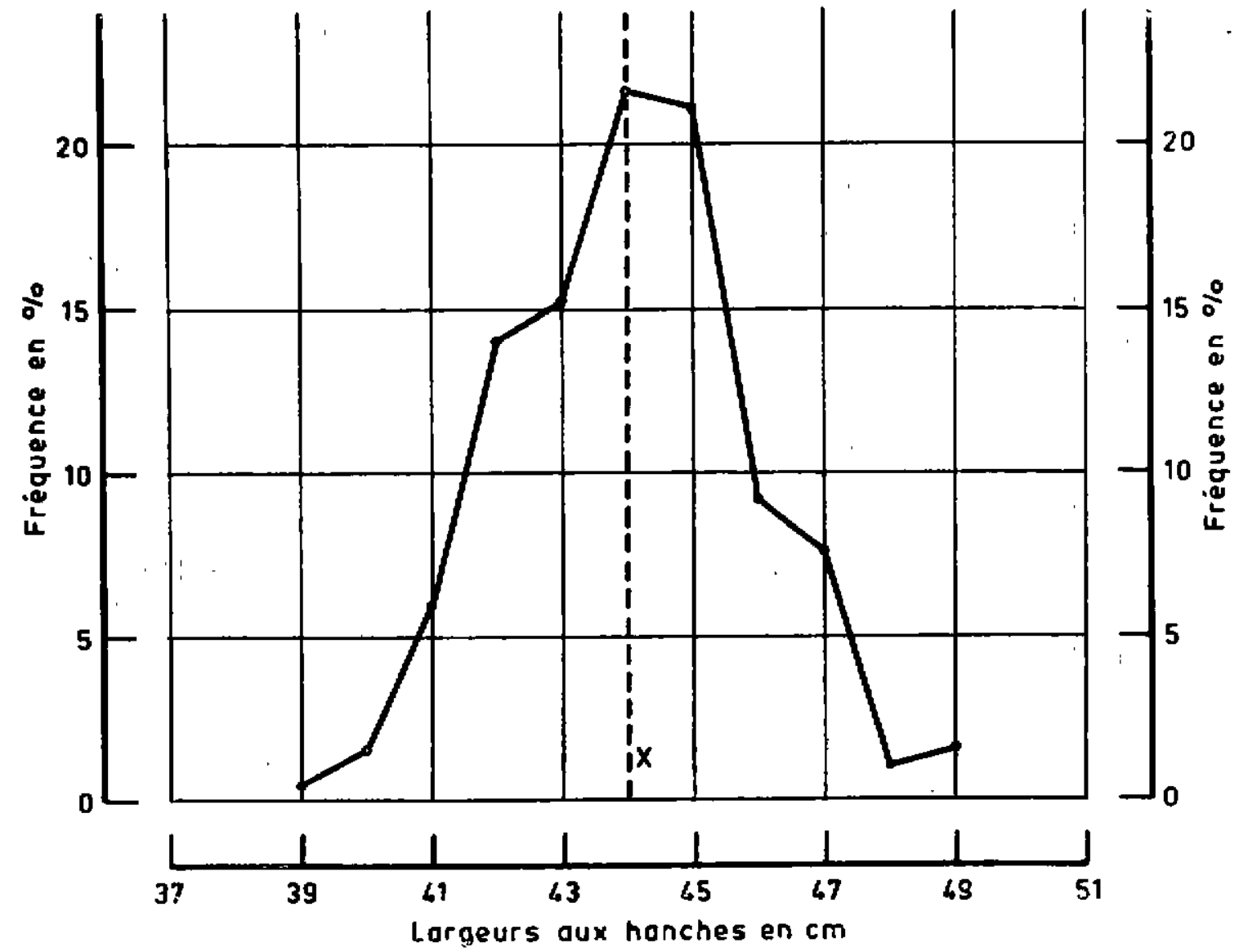

GRAPHIQUE n 10. - Polygone de fréquence des largeurs aux hanches chez les femelles. 
Il est intéressant de déterminer si des animaux hauts profitent également d'une bonne largeur. Le coefficient de corrélation qui exprime la relation entre ces grandeurs, est égal à $0,47 \mathrm{xx}>$ 0,321 (valeur limite au seuil $P=0,001$ ). Les anımaux possédant une bonne hauteur au garrot seront également larges aux hanches.

\section{LES MÂLES.}

Les bœufs extériorisent en moyenne un bassin moins développé que celui des femelles tandis que le bassin des taureaux sélectionnés est nettementiplus long et plus large, surtout en ce qui concerne la largeur d̀ la pointe des fesses.

Les dimensions moyennes sont -

Taureaux Bœufs

Age moyen $\therefore \ldots \ldots \ldots \ldots, 67$ mois 45 mois

21 jours

Largeur aux hanches $\ldots . .4 \quad 46,7 \mathrm{~cm} \quad 41,2 \mathrm{~cm}$

Largeur aux articulations

coxo-fémorales ....... $41,3 \mathrm{~cm} \quad 36,8 \mathrm{~cm}$

Largeur à la pointe des fes-

ses.............. $28,0 \mathrm{~cm} \quad 21,7 \mathrm{~cm}$

Longueur du bassin ...... $51,3 \mathrm{~cm} \quad 46,5 \mathrm{~cm}$

Les équations donnant les dimensions du bassin exprimées en centimètres, en fonction de l'âge en mois, sont :

Pour la largeur aux hanches:

du 1 er au 6 e mois :

$$
\begin{gathered}
\log I_{h}=1,22236+0,03687 A ; \\
I_{h}=16,69 e^{0,08490 A}
\end{gathered}
$$

qu-delà du $12^{\mathrm{e}}$ mois chez les taureaux :

$$
\begin{gathered}
\log I_{p}=1,45879+0,00498 A ; \\
I_{h}=28,76 \mathrm{e}^{\mathbf{0}, 01147 A}
\end{gathered}
$$

Au-delà du $12^{\mathrm{e}}$ mols chez les bceufs :

$$
\begin{gathered}
\log I_{h}=1,43952+0,00411 A ; \\
I_{h}=27,51 \mathrm{e}^{0,00946 \mathrm{~A}}
\end{gathered}
$$

Pour la largeur aux arficulations coxo-fémorales: du 1 er au 6 e mois :

$$
\begin{gathered}
\left.\log \right|_{\mathbf{c} \cdot f}=1,29616+0,02516 \mathrm{~A} ; \\
I_{\mathbf{c} \cdot f}=19,78 \mathrm{e}^{\mathbf{0}, 06793 \mathrm{~A}}
\end{gathered}
$$

Au-delà du $12^{\mathbf{e}}$ mois chez les taureaux :

$$
\begin{gathered}
\text { Log. } I_{\mathrm{c} \cdot \mathrm{f}}=1,46279+0,00350 \mathrm{~A} ; \\
I_{\mathrm{c} \cdot \mathrm{f}}=29,02 \mathrm{e}^{\mathrm{0,00808 \textrm {A }}}
\end{gathered}
$$

Au-delà du $12^{e}$ mois chez les boeufs : $\left.\log \right|_{\mathrm{e} \cdot \mathrm{f}}=1,46351+0,00248 \mathrm{~A}$;

$$
l_{c \cdot p}=29,08 \mathrm{e}^{0,00571 \mathrm{~A}}
$$

Pour lä largeur à la poinfe des fesses : du 1 er au $6^{\text {e mois : }}$

$$
\begin{aligned}
& \text { Log. } I_{p \cdot f}=1,05780+0,02966 A \text {; } \\
& I_{p \cdot p}=11,42 \mathrm{e}^{0,08829 \mathrm{~A}}
\end{aligned}
$$

au-delà du $12 \mathrm{e}$ mois chez les taureaux :

$$
\begin{gathered}
\left.\log \right|_{p \cdot f}=1,20997+0,00528 \mathrm{~A} ; \\
I_{p \cdot f}=16,21 \mathrm{e}^{0,01216 \mathrm{~A}}
\end{gathered}
$$

au-delà du $12^{e}$ mois chez les bœufs :

$$
\begin{gathered}
\left.\log \right|_{p \cdot f}=1,22078+0,00300 \mathrm{~A} ; \\
I_{p \cdot f}=16,63 \mathrm{e}^{0,00011 \mathrm{~A}}
\end{gathered}
$$

Pour lo longueur du bassin :

du 1 er au 6 e mois:

$$
\begin{gathered}
\log L_{b}=1,36454+0,03029 A ; \\
L_{b}=23,15 e^{0,06975 A}
\end{gathered}
$$

au-delà du $12^{\mathbf{e}}$ mois chez les taureaux :

$\log L_{b}=1,54952+0,00386 A$; $\mathrm{L}_{\mathrm{b}}=35,44 \mathrm{e}^{\mathrm{D}, 00889 \mathrm{~A}}$

au-delà du $12^{\mathrm{e}}$ mois chez les boufs :

$$
\begin{gathered}
\log L_{b}=1,51513+0,00351 A ; \\
L_{b}=32,74 e^{0,0080 B_{A}}
\end{gathered}
$$

\section{VII. - CYLINDRE MÉTACARPIEN}

\section{A. Mensuration.}

Le périmètre du canon antérieur. a été pris au niveau du tiers inférieur.

\section{B. Femelles.}

\section{a) Développement.}

La croissance du cylindre métacarpien est pratıquement termınée entre 3 ans et 3 ans et demi. A Mulungu ( $R$.' COMPERE, 1960), la limite de croissance des croisés Bruns-Suisses se situe vers 4 ans.

Les équations donnant le périmètre du métacarpe « $P_{m}$ » en centimètres en fonction de l'âge «A $\mathrm{A}$ en mois, sont :

du 1 er mois au $7 \mathrm{e}$ mois 21 jours :

$$
\begin{gathered}
\log P_{m}=1,08442+0,01382 \mathrm{~A} ; \\
P_{m}=12,15 \mathrm{e}^{0,03182 \mathrm{~A}}
\end{gathered}
$$

du $7 \mathrm{e}$ mois 21 jours au $36 \mathrm{e}$ mols :

$$
\begin{gathered}
\log , P_{m}=1,17652+0,00188, A ; \\
P_{m}=15,02 \mathrm{e}^{0,00493 \mathrm{~A}}
\end{gathered}
$$


b) Valeur moyenne.

La valeur moyenne s'élève à $18 \mathrm{~cm}$. P. MATHIEU (A. FOCAN, 1959) a déterminé d̀ Luvironza une moyenne de $17,2 \mathrm{~cm}$ mais ne spécifie pas l'endroif exact de la mensuration.

TABLEAU NO XIII

Dispersion des données individuelles

\begin{tabular}{|c|c|}
\hline $\begin{array}{c}\text { Périmètre du métacerpe } \\
\text { en cm }\end{array}$ & $\begin{array}{r}\text { Fréquence } \\
\text { en } p \cdot 100\end{array}$ \\
\hline 16 & 2,6 \\
17 & 21,0 \\
18 & 51,1 \\
19 & 19,7 \\
20 & 5,2 \\
21 & 0,4 \\
\hline
\end{tabular}

c) Dispersion des observations.

La dispersion des observations figure au tableau no 13 et au graphique no 11.

Les caractéristiques de l'échantillon observé sont :

Valeur moyenne $\bar{X}=18,0 \mathrm{~cm}$.

Indice de dispers!on $\sigma=0,86 \mathrm{~cm}$.

\section{LES MÂLES.}

Les mâles extérjorisent une ossature plus développée que celle des femelles.' Les valeurs moyennes sont :

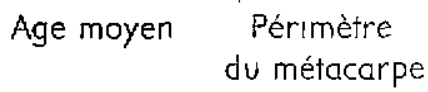

$\begin{array}{llll} & & - & - \\ \text { Taureaux } & \ldots \ldots & 67 \text { mois } & 21,7 \mathrm{~cm} \\ \text { Boufs .......... } & 45 \text { mois } 21 \text { jours } & 20,3 \mathrm{~cm}\end{array}$

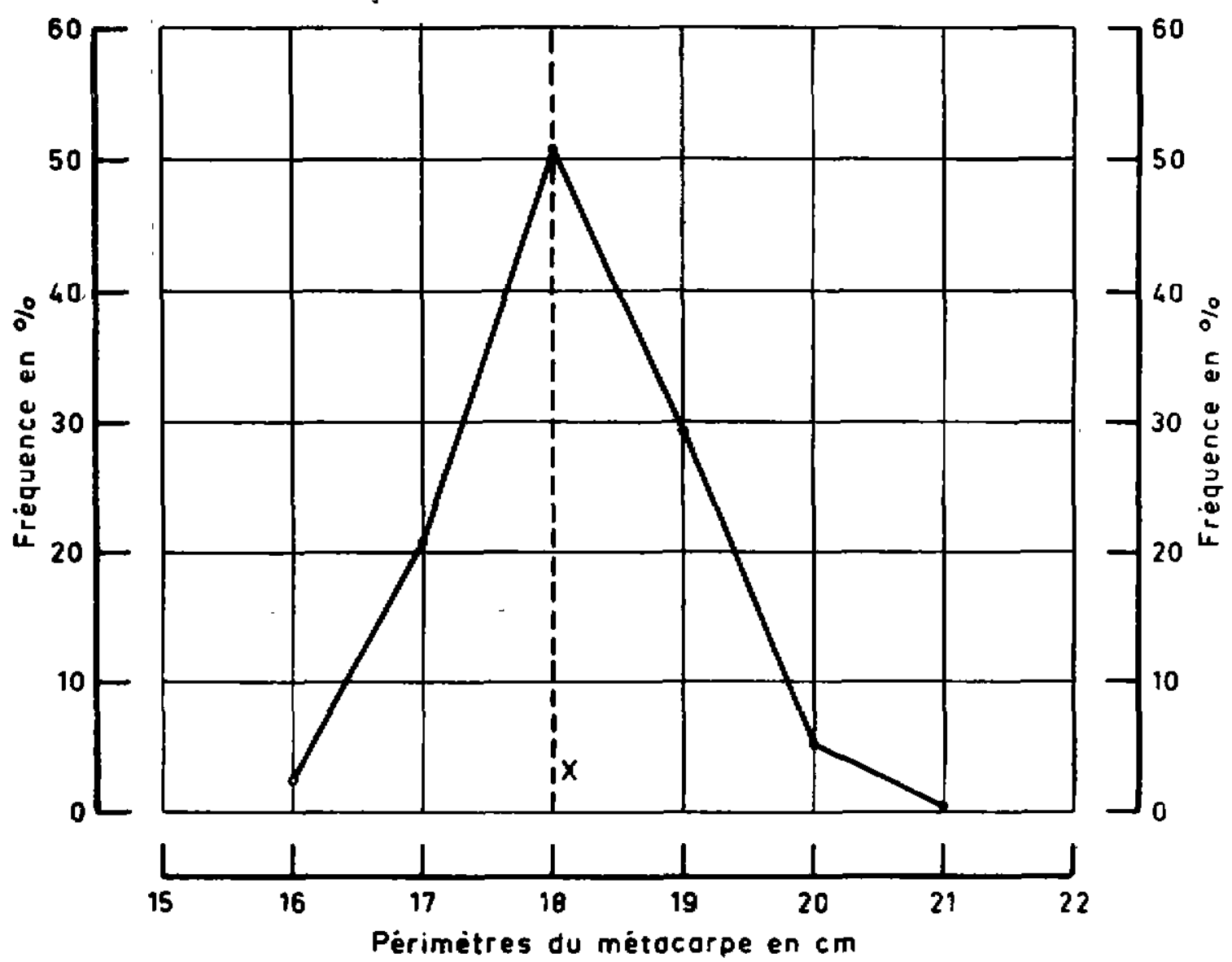

GRAPHIQUE $n^{0}$ 11. - Polygone de fréquence des périmètres du mélacarpe chez ies fernelles. 


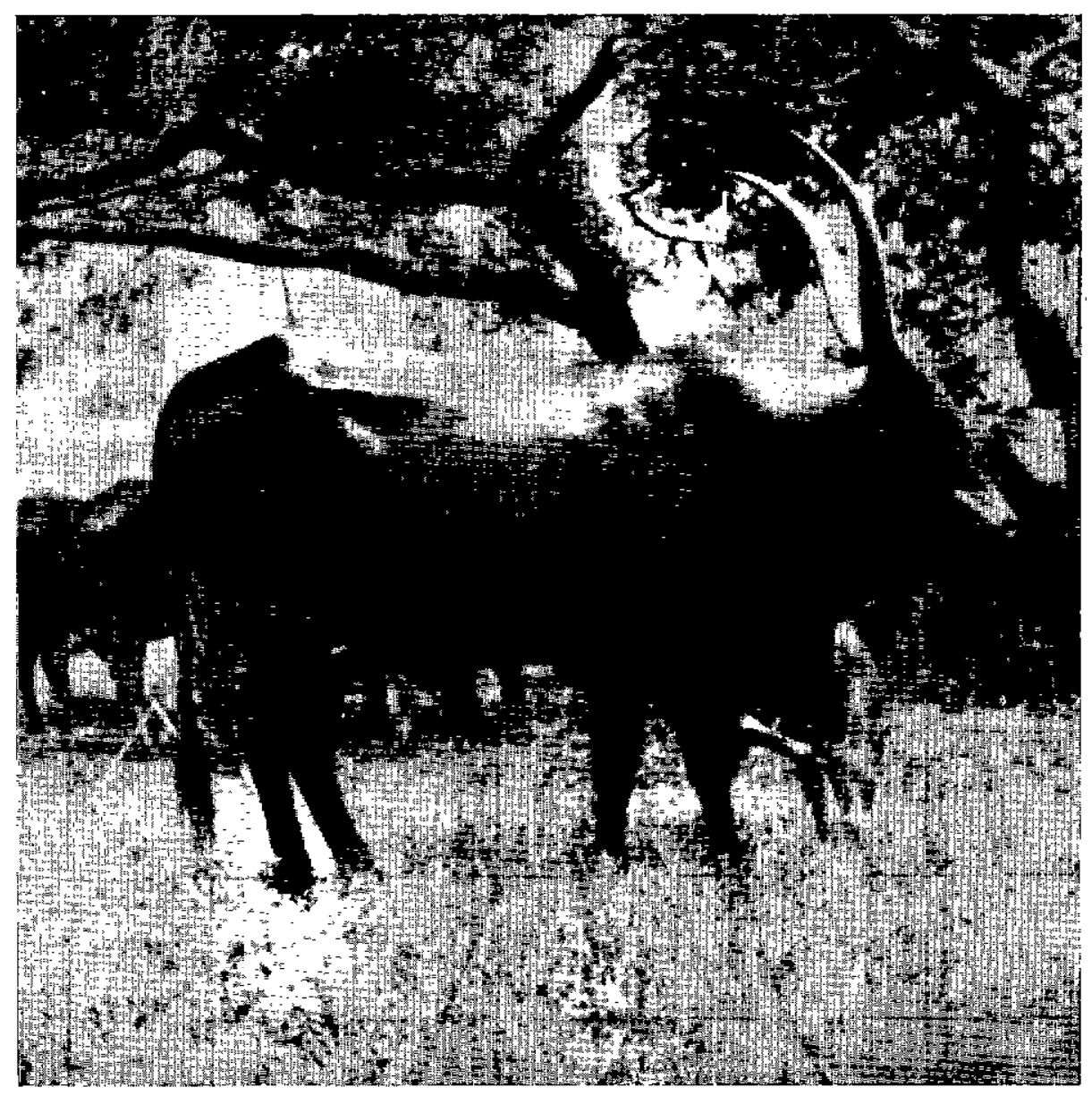

Fig. 1, - Vache $\mathrm{n}^{0}$ 936. Age: 10 ans 7 mois. Poids : $440 \mathrm{~kg}$. Vache de très grand format. 


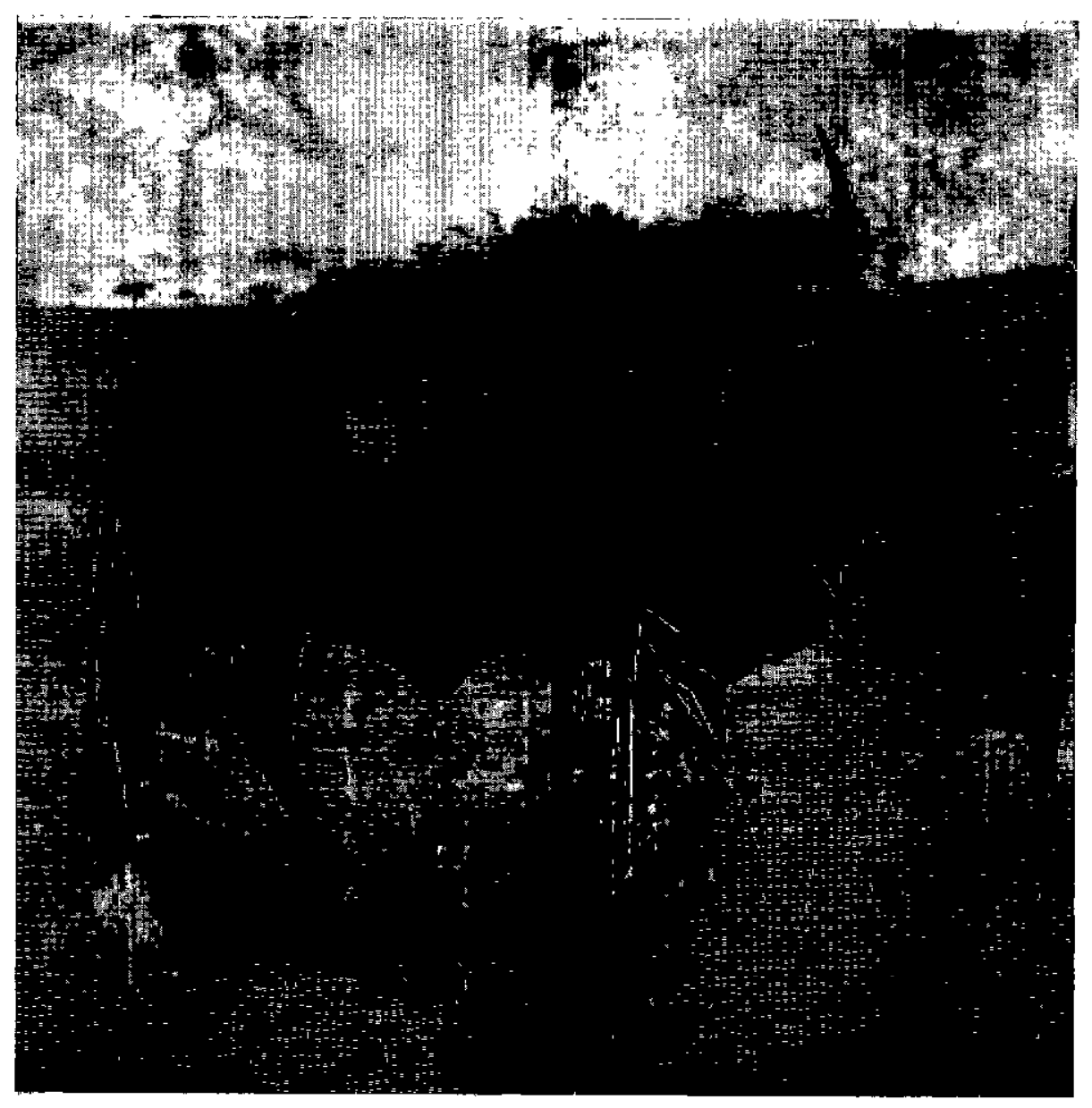

Fig. 2. - Vache no 1514. Age : 7 ans. Poids : $496 \mathrm{~kg}$. 


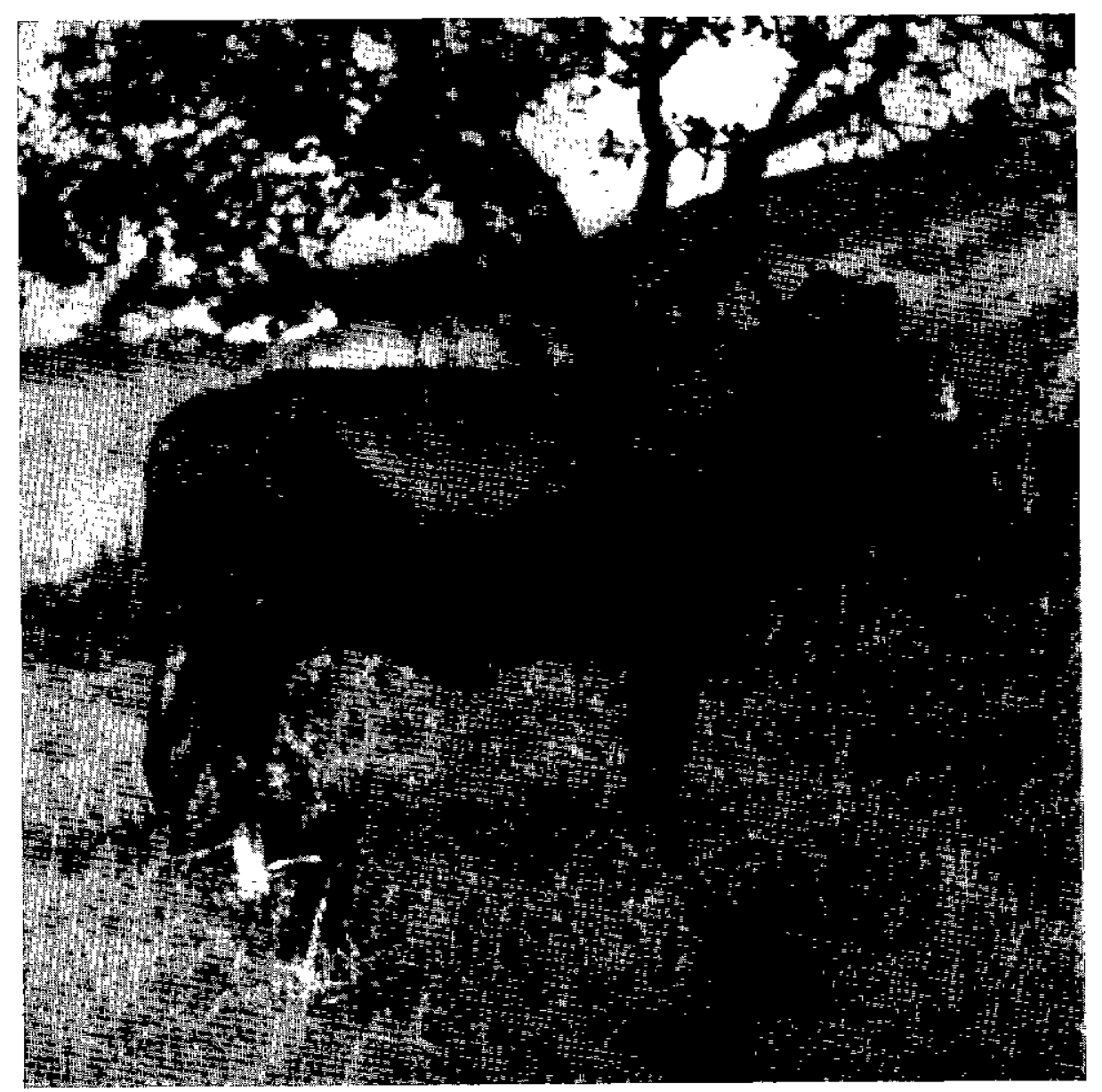

Fig. 3. - Vache no 2222. Age : 3 ans 10 mors. Polds : $383 \mathrm{~kg}$. Animal de bon format. 


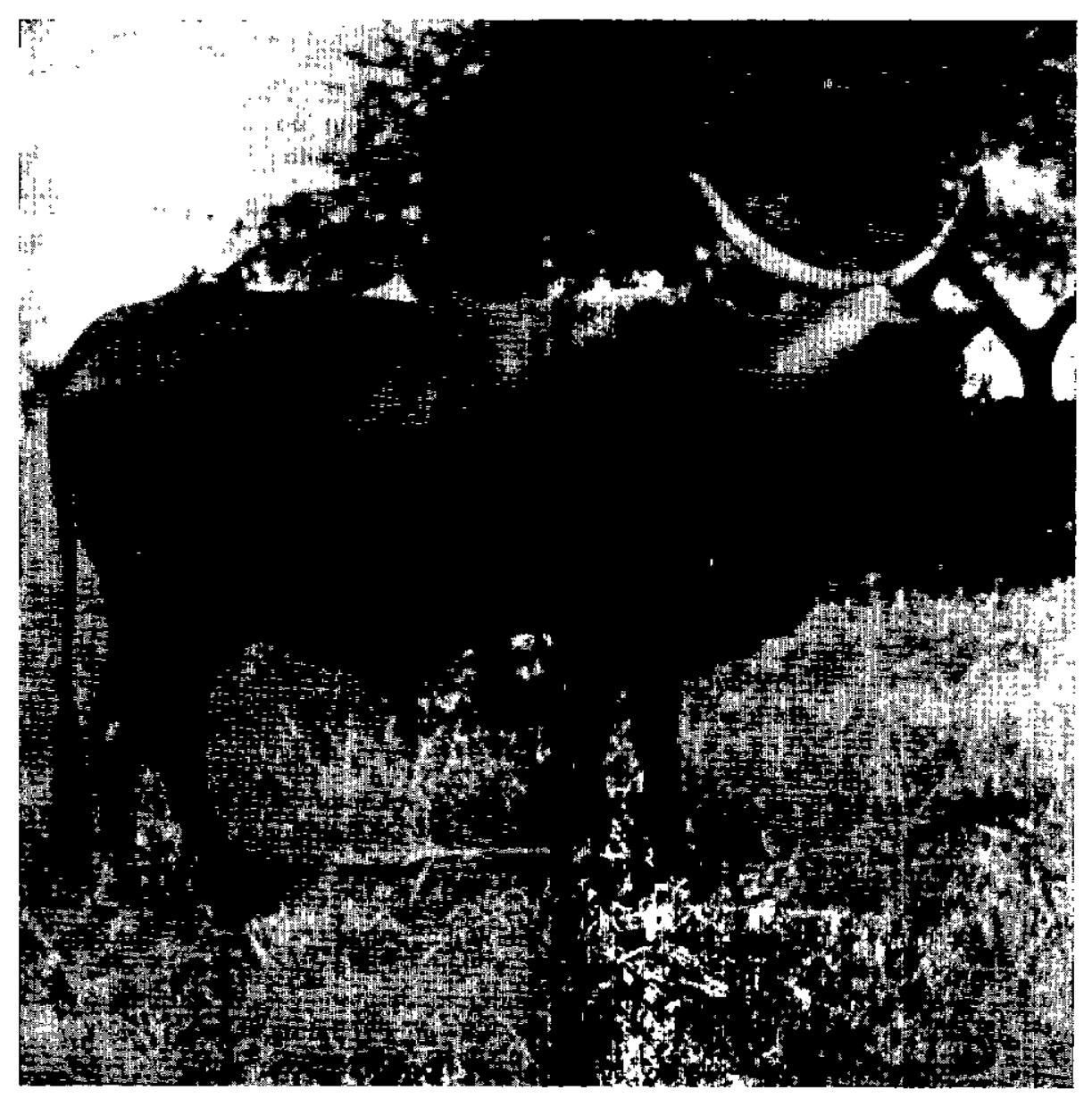

Fig. 4. - Vache $n^{0} 1001$. Age : 9 ans 7 mois. Poids : $401 \mathrm{~kg}$. Animal large. 


\section{CONCLUSIONS}

L'étude approfondie de la conformation du local Nyamiyaga a permis de préciser le rythme de développement des différentes parties du corps d'un tel bétail, de définir le format standard de la population envisagée et de fixer les objectıfs de l'amélioration en ce domaine.

En outre, la recherche de la dispersion des multiples observations et le calcul des corrélations existant entre les mensurations importantes facilitent grandement la détermination des possibilités de l'amélioratıon des diverses caractéristiques de la conformation et permettent la polysélection par le choix de caractères associés.

\title{
SUMMARY
}

\section{Contribution to the study of the conformation of the local Ankole type cattle from Rwanda}

From data callected during the past 25 years of cattle rearing in the Zooiechnic Center of Nyamiyaga (Rwanda Republic), the author has carried out an extensive study of the conformation of the Rwanda Ankole type cattle. Sixteen measurements have been effected on a routine basis with about 400 heads consisting of bulls and cows being in growing period. This work gives precise details on the rhythm of the development of the type of examined cattle. The aims of the improvement of the actual conformation are emphasized: Therefore, the equalon between the living weight and various measurements has been found and interesting correlations have been studied between, the principal dimensions.

\section{RESUMEN}

\section{Contribución al estudio de Ia conformación del ganado local Rwanda del tipo Ankolé}

\begin{abstract}
A partir de datos reunidos durante veinte y cinco anos de crianza en el Centro zootécnico de Nyamiyaga (Republica de Rwanda), el autor hizo un estudio completo de la conformación del ganado de Rwanda del tipo Ankalé.

Hechos en diez y sels medidas tomadas regularmente en casi cuatro cientos animales machos $y$ hembras en pleno periodo de crecimiento, estos trabajos determinan el ritmo de desarollo de las diferentes partes del cuerpo y de sus proporciones, definen el tamano standard del tipo de ganado estudiado $y$ fijan con precisión los objetivos de lá mejoración de la conformación actual. Ademas, se puso en ecuación las relaciones entre el peso vivo y diferentes medidas, y se buscaron correlaciones interesantes entre las principales dimensiones.
\end{abstract}

\section{BIBLIOGRAPHIE}

1948. ABELDOOS, M. - La croissance. 1 vol. 126 p. Presses universitaires de France, Paris.

1961. AURIOL P. ef MOUGIN B. - Production laitière et conformation dans la race Montbeliarde. Annales de Zootechnie, Vol. 10, no 1, p. 5-30.

1958. BLACK W. H., KNAPP J. R., COOK A. C. - Correlation of body measurements of slaughter steers with rate and efficiency of gain and with certain carcass characteristics. J. Agrı. Res. 56, 465.

1958. BLACKMORE D. W. MAC GILLIARD L. D., LUSH J. L. - Relationships between body measurements, meat conformation and milk production. J, Doiry Sc., 41, 1050. 1945. BRODY S. - Bioenergetics and growth. 
1 Vol. Reinhold Publ. Corp. N.Y. (U.S.A.) $1022 \mathrm{pp}$.

1957. Colonial office. The Indigenous Cattle of the British Dependent Territories in Africa. London.

1960. COMPERE R. - Résultats obtenus avec le premier croisement «Bétail indigène $x$ Race Brune des Alpes» à la Station de Mulungu. Bull. Agr. du C. B. et duR. U., Vol. 21, no 3, 617-646.

1951. COOK A. C., KOHLI N. L., DAWSON W. M. - Relationship of five body measurements to slaughter grade, carcass grade, and dressing percentage in Milking Shorthorn steers. J. Anım. Sc. 10, 386.

1959. FOCAN A. - Programme d'élevage au Rwanda-Urundi. Inédit.
1932. LUSH J. L. - The relation of body shape of feeder steers to rate of gain, to dressing per cent and to value of dressed carcass. Texas Agr. Expt. Sta. Bull. 471.

1961. MARICZ M. - Etude comparative des races de zébus et de trors races de bétail de l'Est du Congo. Bull. Agr. du Congo. Vol. L II. no 1, 107-116.

1959. PAGOT J. et DELAINE R. — Etude biométrique de la croissance des taurins N'Dama. Rev. d'Elev. et de Médec. des pays trop. Tome XII, no 4, 405-416.

1953. YAO E. S., DAWSON W. M., COOK A. C. - Relationships between heat production characters and body measurements in beef and Milking Shorthorns. J. Anim. Sc. 12, 775 . 